\title{
More on Generic Dimension Groups
}

\author{
Philip Scowcroft
}

\begin{abstract}
While finitely generic (f.g.) dimension groups are known to admit no proper self-embeddings, these groups also have no automorphisms other than scalar multiplications, and every countable infinitely generic (i.g.) dimension group admits proper self-embeddings and has automorphisms other than scalar multiplications. The finite-forcing companion of the theory of dimension groups is recursively isomorphic to first-order arithmetic, the infinite-forcing companion of the theory of dimension groups is recursively isomorphic to second-order arithmetic, and the first-order theory of existentially closed (e.c.) dimension groups is a complete $\Pi_{1}^{1}$-set. While many special properties of f.g. dimension groups may be realized in recursive e.c. dimension groups, and many special properties of i.g. dimension groups may be realized in hyperarithmetic e.c. dimension groups, no f.g. dimension group is arithmetic and no i.g. dimension group is analytical. Yet there is an f.g. dimension group recursive in first-order arithmetic, and (modulo a set-theoretic hypothesis) there is an i.g. dimension group recursive in second-order arithmetic.
\end{abstract}

\section{Introduction}

A dimension group is a partially ordered abelian group, with directed, ${ }^{1}$ isolated ${ }^{2}$ order, that obeys the Riesz interpolation property. ${ }^{3}$ Countable dimension groups with order unit $^{4}$ provide isomorphism invariants for approximately finite-dimensional (AF) algebras (see Elliott [3]), and Scowcroft [13] shows that one such dimension group is existentially closed in another just in case the corresponding AF algebras, viewed as metric structures (see Ben Yaacov, Berenstein, Henson, and Usvyatsov [2]), fall in the same relation. This model-theoretic correspondence inspired Scowcroft [14], which studied existentially closed (e.c.) dimension groups and revealed special properties of the finitely generic (f.g.) and infinitely generic (i.g.) dimension groups obtained by model-theoretic forcing.

Received March 15, 2012; accepted June 28, 2013

2010 Mathematics Subject Classification: Primary 03C60, 06F20; Secondary 03C25

Keywords: dimension group, finitely generic, infinitely generic, existentially closed

(C) 2015 by University of Notre Dame 10.1215/00294527-3153570 
Motivating the study of these special properties was the hope that sufficiently many of them would characterize f.g. dimension groups among e.c. dimension groups, just as Glass and Pierce [4, Theorem 1.11] and Saracino and Wood [12, Corollary 2.7] show that the f.g. abelian lattice-ordered ( $l$-) groups are exactly the hyperarchimedean e.c. abelian $l$-groups. Sections 2 and 3 below continue this study — with the help of intricate forcing arguments, as in [14, Section 6]—by showing that f.g. dimension groups have only the obvious automorphisms: multiplication by positive rationals. Section 4 reveals an entirely different behavior for countable i.g. dimension groups, which have many more automorphisms as well as proper self-embeddings. Yet Section 5 begins to show that f.g. dimension groups, despite these very special algebraic properties, behave very differently from f.g. abelian $l$-groups. While some of these are recursive-have recursive diagram-and all share the same recursive first-order theory, definability results from [14, Section 6] may be extended to define copies of the rational field within e.c. dimension groups, to show that the finite-forcing companion of the theory of dimension groups is recursively isomorphic to first-order arithmetic, and to show that no f.g. dimension group is arithmetic (i.e., has diagram definable in first-order arithmetic). Section 6 presents parallel results for i.g. dimension groups: the infinite-forcing companion is recursively isomorphic to second-order arithmetic, and no i.g. dimension group is analytical (i.e., has diagram definable in second-order arithmetic). Confirming the doubts raised by Section 5, Section 7 shows that recursive e.c. dimension groups may satisfy all of the special properties of f.g. dimension groups discovered in [14] and in Sections 2 and 3; so these striking properties fall far short of characterizing f.g. dimension groups, which cannot be arithmetic though they can be recursive in first-order arithmetic. Once again, i.g. dimension groups obey parallel results at a higher level of recursion-theoretic complexity, and Section 8 shows that hyperarithmetic e.c. dimension groups may satisfy all of the special properties of i.g. dimension groups discovered in [14] and in Section 4, and that (modulo a set-theoretic hypothesis) there is an i.g. dimension group recursive in second-order arithmetic. After Section 9 mentions some open problems, an Appendix establishes technical results for various relations of forcing on which some of the arguments in Sections 5-8 rely.

Readers may wish to refer to [14] as needed, especially to its Section 6, which (through [14, Corollary 6.26]) presents helpful results on definability in e.c. dimension groups as well as techniques useful in finite-forcing arguments. Model-theoretic notions not explained here may be found in Hirschfeld and Wheeler [6] and Hodges [7], [8].

\section{Automorphisms and Positive Elements in f.g. Dimension Groups}

Because e.c. dimension groups are divisible and both f.g. and i.g. dimension groups are e.c., one may restrict attention to divisible dimension groups in what follows. So dimension groups will be viewed as structures for the language $\mathcal{Q}=\{+,-,<, 0\} \cup\{r \cdot: r \in \mathbb{Q}\}$ of divisible partially ordered abelian groups. Let $T^{\mathcal{Q}}$ be the $\mathcal{Q}$-theory of divisible dimension groups, and let $W$ be the countable set of witnesses. ${ }^{5}$ In what follows, $t \mid v$ will abbreviate

$$
t \nless v \wedge t \neq v \wedge v \nless t \text {. }
$$

Several theorems of this paper rest upon the following result about forcing, which in this and the next section is finite forcing. 
Lemma 2.1 Let $\gamma \in \mathbb{R}$, and let $f: \mathbb{R}^{2} \rightarrow \mathbb{R}$ be strictly increasing in the first argument and continuous. If $v$ and $w$ are distinct witnesses, then $\{v>0\}$ forces

$\bigvee_{k \in \mathbb{Q}} w=k v \vee \bigvee_{k<l \in \mathbb{Q}, f(k, l)<\gamma}(k v \mid w \wedge w<l v) \vee \bigvee_{k<l \in \mathbb{Q}, f(k, l)>\gamma}(k v<w \wedge l v \mid w)$

Proof Given a condition $p \supseteq\{v>0\}$, one wants to find a condition $q \supseteq p$ forcing one of the displayed disjuncts. $p$ may be satisfied in some $\mathbb{Q}$-power $\mathbb{Q}^{n}$ (see $\left[14\right.$, Lemma 6.3]), and one may assume that $w$ is not a scalar multiple of $v$ in $\mathbb{Q}^{n}$. So if

$$
r_{\alpha}=\operatorname{lub}\left\{s \in \mathbb{Q}: s v<w \text { in } \mathbb{Q}^{n}\right\}
$$

and

$$
r_{\beta}=\operatorname{glb}\left\{s \in \mathbb{Q}: w<s v \text { in } \mathbb{Q}^{n}\right\},
$$

then $r_{\alpha}<r_{\beta}$ in $\mathbb{Q}$ and $t v \mid w$ in $\mathbb{Q}^{n}$ for all rational $t \in\left[r_{\alpha}, r_{\beta}\right]$.

Suppose that $f\left(r_{\alpha}, r_{\beta}\right) \neq \gamma$. If $f\left(r_{\alpha}, r_{\beta}\right)<\gamma$, then since $f$ is continuous there is $l>r_{\beta}$ in $\mathbb{Q}$ with $f\left(r_{\alpha}, l\right)<\gamma$, and

$$
r_{\alpha} v \mid w \wedge w<l v
$$

in $\mathbb{Q}^{n}$. A similar argument shows that if $f\left(r_{\alpha}, r_{\beta}\right)>\gamma$, then there is $k<r_{\alpha}$ in $\mathbb{Q}$ with $f\left(k, r_{\beta}\right)>\gamma$, and

$$
k v<w \wedge r_{\beta} v \mid w
$$

in $\mathbb{Q}^{n}$.

Assume now that $f\left(r_{\alpha}, r_{\beta}\right)=\gamma$. The proof of Theorem 6.18 in [14], with $r=r_{\alpha}$, shows that the assignment in $\mathbb{Q}^{n}$ may be converted to an assignment in $\mathbb{Q}^{n+2}$ that satisfies $p \cup\{s v \mid w\}$ for some $s<r_{\alpha}$ without changing

$$
r_{\beta}=\operatorname{glb}\left\{h \in \mathbb{Q}: w<h v \text { in } \mathbb{Q}^{n+2}\right\}
$$

(in the earlier proof pick $t<r_{\beta}$ ). Since $f$ is strictly increasing in its first argument, $f\left(s, r_{\beta}\right)<\gamma$; and since $f$ is continuous, there is $l>r_{\beta}$ in $\mathbb{Q}$ with $f(s, l)<\gamma$. So

$$
s v \mid w \wedge w<l v
$$

in $\mathbb{Q}^{n+2}$, and one may let $q=p \cup\{s v \mid w, w<l v\}$.

Lemma 6.21 of [14] provides an $\exists \forall$-formula-written “ $y \in \mathbb{Q} \cdot x$ ” in what followswhich defines $\mathbb{Q} \cdot a$ in any e.c. dimension group to which $a$ belongs. Because all f.g. dimension groups are e.c., one may combine this definability result with Lemma 2.1-applied to the addition function $f$-to conclude the following.

Corollary 2.2 If $r \in \mathbb{Q}$, then in any f.g. dimension group

$$
\begin{aligned}
\forall x & >0 \forall y(y \notin \mathbb{Q} \cdot x \rightarrow \exists k, l \in \mathbb{Q} \cdot x(k<l \\
& \wedge[(k+l<r x \wedge k \mid y \wedge y<l) \\
& \vee(k+l>r x \wedge k<y \wedge l \mid y)])) .
\end{aligned}
$$

One thus reaches the following conclusion.

Theorem 2.3 In any f.g. dimension group

$$
\begin{aligned}
& \forall x>0 \forall y, y^{\prime}\left(\forall z \in \mathbb { Q } \cdot x \left[\left(z<y \leftrightarrow z<y^{\prime}\right)\right.\right. \\
& \left.\left.\quad \wedge\left(z|y \leftrightarrow z| y^{\prime}\right) \wedge\left(y<z \leftrightarrow y^{\prime}<z\right)\right] \rightarrow y=y^{\prime}\right) .
\end{aligned}
$$


Proof Assume that in the f.g. dimension group $\mathcal{E}, a>0$ and

$$
\left(t a<b \leftrightarrow t a<b^{\prime}\right) \wedge\left(t a|b \leftrightarrow t a| b^{\prime}\right) \wedge\left(b<t a \leftrightarrow b^{\prime}<t a\right)
$$

for all $t \in \mathbb{Q}$.

If $b=t a$ for some $t \in \mathbb{Q}$ and $b^{\prime} \neq t a$, then either $b^{\prime}=s a$ for some $s \neq t$ in $\mathbb{Q}$ or $b^{\prime} \notin \mathbb{Q} \cdot a$. In the first case

$$
s<t \Rightarrow b \nless t a \wedge b^{\prime}<t a
$$

and

$$
t<s \Rightarrow t a \nless b \wedge t a<b^{\prime} ;
$$

so the second case occurs, and

$$
\begin{aligned}
& b^{\prime}<t a \Rightarrow b \nless t a \wedge b^{\prime}<t a, \\
& t a<b^{\prime} \Rightarrow t a \nless b \wedge t a<b^{\prime},
\end{aligned}
$$

and

$$
t a\left|b^{\prime} \Rightarrow t a \nmid b \wedge t a\right| b^{\prime} .
$$

Thus $b=b^{\prime}$ if $b \in \mathbb{Q} \cdot a$, and a symmetric argument shows that $b=b^{\prime}$ if $b^{\prime} \in \mathbb{Q} \cdot a$.

Assume now that $b, b^{\prime} \notin \mathbb{Q} \cdot a$. One sees that $(a, b)$ and $\left(a, b^{\prime}\right)$ have the same quantifier-free type, which by [14, Theorem 6.18] is determined by $\alpha<\beta$ in $\mathbb{R} \backslash \mathbb{Q}$ such that for all $t \in \mathbb{Q}$,

$$
\begin{aligned}
& \left(t<\alpha \text { iff } t a<b, b^{\prime}\right) \quad \text { and } \quad\left(\alpha<t<\beta \text { iff } t a \mid b, b^{\prime}\right) \quad \text { and } \\
& \left(\beta<t \text { iff } b, b^{\prime}<t a\right) .
\end{aligned}
$$

Theorem 6.25 and Corollary 6.26 of [14] imply that $b$ is in the linear span of $a, b^{\prime}$ or that $a, b, b^{\prime}$ are linearly dependent. Because neither $b$ nor $b^{\prime}$ is a scalar multiple of $a$, there are rational $r$ and $s$ with $b^{\prime}=s b+r a$ and $s \neq 0$.

Suppose that $s>0$. For $t \in \mathbb{Q}$,

$$
\begin{array}{lll}
t<\alpha & \text { iff } & t a<b^{\prime} \\
& \text { iff } & t a<s b+r a \\
& \text { iff } & \frac{t-r}{s} a<b \\
& \text { iff } & \frac{t-r}{s}<\alpha \\
\text { iff } & t<s \alpha+r ;
\end{array}
$$

so $\alpha=s \alpha+r,(1-s) \alpha=r \in \mathbb{Q}, s=1$ because $\alpha$ is irrational, and $r=0$. Thus $b=b^{\prime}$ if $s>0$.

Now suppose that $s<0$. For $t \in \mathbb{Q}$, arguments like that in the last paragraph show that

$$
t<\alpha \quad \text { iff } \quad t<s \beta+r
$$

and that

$$
\beta<t \quad \text { iff } \quad s \alpha+r<t .
$$

So $\alpha=s \beta+r$ and $\beta=s \alpha+r$, and

$$
\alpha=s(s \alpha+r)+r=s^{2} \alpha+s r+r .
$$

$s^{2}=1$ because $\alpha$ is irrational; so since $s<0, s=-1$ and $\alpha+\beta=r$. Corollary 2.2 now provides $k<l$ in $\mathbb{Q}$ such that either $k+l<r$ and

$$
k a \mid b \wedge b<l a
$$


or $k+l>r$ and

$$
k a<b \wedge l a \mid b .
$$

In the first case $k+l<r, \alpha<k<\beta$, and $\beta<l$; so $\alpha+\beta<k+l<r=\alpha+\beta$. Thus the second case holds, and $k+l>r, k<\alpha$, and $\alpha<l<\beta$; but now $r<k+l<\alpha+\beta=r$. This final contradiction implies that $s \nless 0$, and so the previous argument completes the proof.

One thus reaches the following conclusion.

Corollary 2.4 If $v>0$ in the f.g. dimension group $\mathcal{E}$, then any automorphism $\varphi$ of $\mathcal{G}$ is determined by $\varphi(v)$.

Proof One need show merely that if $\varphi(v)=v$, then $\varphi$ is the identity map. If $w$ is any element of $G$, then for any $t \in \mathbb{Q}$,

$$
(t v<w \leftrightarrow t v<\varphi(w)) \wedge(t v|w \leftrightarrow t v| \varphi(w)) \wedge(w<t v \leftrightarrow \varphi(w)<t v)
$$

because $\varphi(v)=v$; so $\varphi(w)=w$ by Theorem 2.3.

\section{The Group of Automorphisms of an f.g. Dimension Group}

One may reach a much stronger conclusion about automorphisms with the help of the following lemma.

Lemma 3.1 If $v, v^{\prime}, w, w^{\prime}$ are distinct witnesses, then $\left\{v, v^{\prime}>0\right\}$ forces

$$
\begin{aligned}
\bigvee_{\overline{0} \neq \bar{q} \in \mathbb{Q}^{3}} q_{1} v+q_{2} v^{\prime}+q_{3} w= & \vee \\
& \vee \bigvee_{r<0} w<r v \vee \bigvee_{0<r} r v<w \\
& \vee \neg\left(w<r v \leftrightarrow w^{\prime}<r v^{\prime}\right) \\
& \vee \bigvee_{r \in \mathbb{Q}} \neg\left(r v<w \leftrightarrow r v^{\prime}<w^{\prime}\right) .
\end{aligned}
$$

Proof If the condition $p$ extends $\left\{v, v^{\prime}>0\right\}$, one wants to find an extension of $p$ that forces one of the displayed disjuncts. $p$ may be satisfied by an assignment $\rho$ in some $\mathbb{Q}^{n}$, and without loss of generality $v, v^{\prime}, w$ are linearly independent in $\mathbb{Q}^{n}$. Any identity in $p$ involving a witness other than $v, v^{\prime}, w$, and $w^{\prime}$ may be used to eliminate that witness from $p$, and the resulting condition $q$ still is satisfied by $\rho$ and has the property that for any displayed disjunct $\delta, p \cup\{\delta\}$ is satisfiable when $q \cup\{\delta\}$ is satisfiable. One may therefore assume that $p$ contains no identities involving witnesses other than $v, v^{\prime}, w$, and $w^{\prime}$. Since $v, v^{\prime}$, and $w$ are linearly independent in $\mathbb{Q}^{n}$, one may assume that either $v, v^{\prime}, w$, and $w^{\prime}$ are linearly independent in $\mathbb{Q}^{n}$ or that $p$ contains exactly one identity, which contains $w^{\prime}$ nontrivially.

Suppose first that $v, v^{\prime}, w$, and $w^{\prime}$ are linearly independent in $\mathbb{Q}^{n}$. One may assume that $p$ contains neither identities nor formulas $g \nless g$; and by replacing each formula $g \neq h$ or $g \nless h$ in $p$ by the stronger formula

$$
g<h, \quad h<g, \quad \text { or } \quad h \mid g
$$

true in $\mathbb{Q}^{n}$, one may assume that $p$ consists of inequalities and incomparabilities. Each incomparability that contains $v$ nontrivially is equivalent modulo $T^{\mathcal{Q}}$ to a formula

$$
v \mid t
$$


where $t$ is a closed $\mathcal{Q}_{W}$-term not containing $v$; and one may assume that incomparabilities in $p$ either do not contain $v$ or are of the form displayed. Let $\varphi=\varphi(v, \ldots)$ be the conjunction of all inequalities in $p$. Because $v>0$ in $\mathbb{Q}^{n}$ and $\varphi$ consists of inequalities, there is $r_{0} \in(0,1) \cap \mathbb{Q}$ such that for all $t \in\left(-r_{0}, r_{0}\right) \cap \mathbb{Q}$,

$$
\varphi((1+t) v, \ldots) \text { in } \mathbb{Q}^{n} .
$$

Each incomparability ( $\Delta)$ in $p$ is true in $\mathbb{Q}^{n}$ either because $v-t$ has both positive and negative coordinates in $\mathbb{Q}^{n}$ or because the coordinates of $v-t$ in $\mathbb{Q}^{n}$ are always nonnegative (nonpositive) but not always zero and not always positive (negative). Calling all incomparabilities of the second kind singular, one may proceed as in [14, Section 6] to eliminate singular incomparabilities by changing the assignment in $\mathbb{Q}^{n}$. So let

$$
v\left|t_{1}, \ldots, v\right| t_{q}
$$

be all the singular incomparabilities in $p$. Copying $\rho q+1$ times, one obtains an assignment $\sigma$ in $\mathbb{Q}^{n(q+1)}$ that satisfies $p$ as well as $\varphi((1+t) v, \ldots)$ whenever $t \in\left(-r_{0}, r_{0}\right) \cap \mathbb{Q}$. One now changes $\sigma$ to an assignment $\tau$ in $\mathbb{Q}^{n(q+1)}$ that agrees with $\sigma$ on all witnesses but $v$. For $i=1, \ldots, q$ the incomparability $v \mid t_{i}$ is singular with respect to $\rho$, and so there is $j_{i}$ with $1 \leq j_{i} \leq n$ and

$$
\rho(v)_{j_{i}}=\rho\left(t_{i}\right)_{j_{i}} .
$$

$\tau(v)$ results from $\sigma(v)$ when it is changed just at the coordinates $n i+j_{i}$ with $1 \leq i \leq q$, and

$$
\tau(v)_{n i+j_{i}}=\rho\left(t_{i}\right)_{j_{i}} \pm(1 / 2) r_{0} \rho(v)_{j_{i}},
$$

where the sign is $-(+)$ when all coordinates of $\rho\left(v-t_{i}\right)$ are nonnegative (nonpositive). Every incomparability $v \mid t$ in $p$ is nonsingular with respect to $\tau$, which still satisfies $p$ by the choice of $r_{0}$. There is a positive rational $r_{1} \leq r_{0}$ such that for all $s \in\left(-r_{1}, r_{1}\right) \cap \mathbb{Q}$ and all incomparabilities $v \mid t$ in $p, \tau$ satisfies $\varphi((1+s) v, \ldots)$ and

$$
\tau(v-t)_{i} \neq 0 \Rightarrow \tau((1+s) v-t)_{i} \text { and } \tau(v-t)_{i} \text { have the same sign. }
$$

So $\tau$ satisfies $p(v /(1+s) v)$ for all $s \in\left(-r_{1}, r_{1}\right) \cap \mathbb{Q}$, where $p(v /(1+s) v)$ results from $p$ when all occurrences of $v$ are replaced by $(1+s) v$.

Since $v$ and $w$ are linearly independent in $\mathbb{Q}^{n(q+1)}$, there are $r_{\alpha}<r_{\beta}$ in $\mathbb{Q}$ with

$$
r_{\alpha}=\operatorname{lub}\left\{r \in \mathbb{Q}: r v<w \text { in } \mathbb{Q}^{n(q+1)}\right\}
$$

and

$$
r_{\beta}=\operatorname{glb}\left\{r \in \mathbb{Q}: w<r v \text { in } \mathbb{Q}^{n(q+1)}\right\} .
$$

Since $v^{\prime}$ and $w^{\prime}$ are linearly independent in $\mathbb{Q}^{n(q+1)}$, there are $r_{\alpha}^{\prime}<r_{\beta}^{\prime}$ in $\mathbb{Q}$ with

$$
r_{\alpha}^{\prime}=\operatorname{lub}\left\{r \in \mathbb{Q}: r v^{\prime}<w^{\prime} \text { in } \mathbb{Q}^{n(q+1)}\right\}
$$

and

$$
r_{\beta}^{\prime}=\operatorname{glb}\left\{r \in \mathbb{Q}: w^{\prime}<r v^{\prime} \text { in } \mathbb{Q}^{n(q+1)}\right\} .
$$

If $r_{\alpha} \neq r_{\alpha}^{\prime}$ or $r_{\beta} \neq r_{\beta}^{\prime}$ - say $r_{\alpha}<r_{\alpha}^{\prime}$-then in $\mathbb{Q}^{n(q+1)}$,

$$
r_{\alpha} v \mid w \wedge r_{\alpha} v^{\prime}<w^{\prime}
$$


and the argument is complete; so assume that $r_{\alpha}=r_{\alpha}^{\prime}$ and $r_{\beta}=r_{\beta}^{\prime}$. Since $r_{\alpha}<r_{\beta}$, $r_{\beta}>0$ or $r_{\alpha}<0$. If $r_{\beta}>0$, change $\tau$ to $\gamma$ by letting $\gamma \uparrow W \backslash\{v\}=\tau \uparrow W \backslash\{v\}$ and

$$
\gamma(v)=\tau\left(\left(1+(1 / 2) r_{1}\right) v\right) .
$$

The choice of $r_{1}$ implies that $\gamma$ satisfies $p$, and since $\gamma \uparrow\left\{v^{\prime}, w^{\prime}\right\}=\tau \uparrow\left\{v^{\prime}, w^{\prime}\right\}$,

$$
\operatorname{glb}\left\{r \in \mathbb{Q}: \gamma \text { makes } w^{\prime}<r v^{\prime} \text { in } \mathbb{Q}^{n(q+1)}\right\}=r_{\beta}^{\prime}=r_{\beta} .
$$

Because

$$
r_{\beta}<r \quad \text { iff } \quad \tau \text { makes } w<r v
$$

for all $r \in \mathbb{Q}$, and $1+(1 / 2) r_{1}>0$,

$\frac{r_{\beta}}{1+(1 / 2) r_{1}}<\frac{r}{1+(1 / 2) r_{1}} \quad$ iff $\quad \tau$ makes $w<\frac{r}{1+(1 / 2) r_{1}}\left(1+(1 / 2) r_{1}\right) v$.

So because $\gamma(w)=\tau(w)$ and $\gamma(v)=\tau\left(\left(1+(1 / 2) r_{1}\right) v\right)$,

$$
\begin{array}{lll}
\frac{r_{\beta}}{1+(1 / 2) r_{1}}<s & \text { iff } & \tau \text { makes } w<s\left(1+(1 / 2) r_{1}\right) v \\
& \text { iff } \quad \gamma \text { makes } w<s v
\end{array}
$$

for all $s \in \mathbb{Q}$. Since $r_{\beta}>0$ and $1+(1 / 2) r_{1}>1$,

$$
r_{\beta}>\frac{r_{\beta}}{1+(1 / 2) r_{1}}=\operatorname{glb}\{s \in \mathbb{Q}: \gamma \text { makes } w<s v\} .
$$

Thus $\gamma$ satisfies

$$
w<r_{\beta} v \wedge w^{\prime} \mid r_{\beta} v^{\prime}
$$

and the argument is complete if $r_{\beta}>0$. If $r_{\alpha}<0$, one may apply the previous argument to $(v,-w)$ and $\left(v^{\prime},-w^{\prime}\right)$, for which the numbers corresponding to $r_{\alpha}$ and $r_{\beta}$ are $-r_{\beta}$ and $-r_{\alpha}$.

Suppose now that $p$ contains an identity. Since it contains $w^{\prime}$ nontrivially, the identity is equivalent modulo $T^{\mathcal{Q}}$ to an identity

$$
w^{\prime}=d w+e v+f v^{\prime} .
$$

One may use it to eliminate $w^{\prime}$ from $p$ to get $p^{*}$; and $p \cup\left\{\neg\left(w<r v \leftrightarrow w^{\prime}<r v^{\prime}\right)\right\}$ $\left(p \cup\left\{\neg\left(r v<w \leftrightarrow r v^{\prime}<w^{\prime}\right)\right\}, p \cup\{w<r v\}, p \cup\{r v<w\}\right)$ is consistent with $T^{\mathcal{Q}}$ whenever $p^{*} \cup\left\{\neg\left(w<r v \leftrightarrow d w+e v+f v^{\prime}<r v^{\prime}\right)\right\}$ $\left(p^{*} \cup\left\{\neg\left(r v<w \leftrightarrow r v^{\prime}<d w+e v+f v^{\prime}\right)\right\}, p^{*} \cup\{w<r v\}, p^{*} \cup\{r v<w\}\right)$ is consistent with $T^{\mathcal{Q}}$. As above one may assume that $p^{*}$ consists only of inequalities and incomparabilities, and one may find an assignment $\tau$ in some $\mathbb{Q}^{n(q+1)}$ that satisfies $p^{*}$, that makes all incomparabilities involving $v$ nonsingular, and for which there is $r_{1} \in(0,1) \cap \mathbb{Q}$ such that for all $s \in\left(-r_{1}, r_{1}\right) \cap \mathbb{Q}, \tau$ satisfies $p^{*}(v /(1+s) v)$. Since $v$ and $w$ are linearly independent in $\mathbb{Q}^{n(q+1)}$, there are $r_{\alpha}<r_{\beta}$ in $\mathbb{Q}$ with

$$
r_{\alpha}=\operatorname{lub}\left\{r \in \mathbb{Q}: r v<w \text { in } \mathbb{Q}^{n(q+1)}\right\}
$$

and

$$
r_{\beta}=\operatorname{glb}\left\{r \in \mathbb{Q}: w<r v \text { in } \mathbb{Q}^{n(q+1)}\right\} .
$$


If there is $g \in \mathbb{Q}$ with $w^{\prime}=g v^{\prime}$ in $\mathbb{Q}^{n(q+1)}$ — that is, $d w+e v+f v^{\prime}=g v^{\prime}$ in $\mathbb{Q}^{n(q+1)}$-then

$$
\begin{aligned}
g<r_{\alpha} & \Rightarrow g v<w \wedge g v^{\prime} \nless w^{\prime} \quad \text { in } \mathbb{Q}^{n(q+1)}, \\
r_{\beta}<g & \Rightarrow w<g v \wedge w^{\prime} \nless g v^{\prime} \quad \text { in } \mathbb{Q}^{n(q+1)}, \\
r_{\alpha}<g \leq r_{\beta} & \Rightarrow r_{\alpha} v \nless w \wedge r_{\alpha} v^{\prime}<w^{\prime} \quad \text { in } \mathbb{Q}^{n(q+1)}, \\
g=r_{\alpha} \Rightarrow w \nless r_{\beta} v \wedge w^{\prime}<r_{\beta} v^{\prime} & \text { in } \mathbb{Q}^{n(q+1)} .
\end{aligned}
$$

If there is no such $g$, then $v^{\prime}$ and $w^{\prime}$ are linearly independent in $\mathbb{Q}^{n(q+1)}$, and there are $r_{\alpha}^{\prime}<r_{\beta}^{\prime}$ in $\mathbb{Q}$ with

$$
r_{\alpha}^{\prime}=\operatorname{lub}\left\{r \in \mathbb{Q}: r v^{\prime}<w^{\prime} \text { in } \mathbb{Q}^{n(q+1)}\right\}
$$

and

$$
r_{\beta}^{\prime}=\operatorname{glb}\left\{r \in \mathbb{Q}: w^{\prime}<r v^{\prime} \text { in } \mathbb{Q}^{n(q+1)}\right\} .
$$

If $r_{\alpha} \neq r_{\alpha}^{\prime}$ or $r_{\beta} \neq r_{\beta}^{\prime}$, then one reaches the desired conclusion as before; so assume that $r_{\alpha}=r_{\alpha}^{\prime}$ and $r_{\beta}=r_{\beta}^{\prime}$.

If $e=0$, then the argument handling linearly independent $v, v^{\prime}, w, w^{\prime}$ gives the desired conclusion, since changing $\tau(v)$ to $\gamma(v)=\tau\left(\left(1+(1 / 2) r_{1}\right) v\right)$ does not change the value of $d w+f v^{\prime}=w^{\prime}$.

Assume now that $e>0$. If $r_{\beta}<0$, then $\tau$ satisfies $p^{*} \cup\left\{w<(1 / 2) r_{\beta} v\right\}$, where $(1 / 2) r_{\beta}<0$; so assume that $r_{\beta} \geq 0$. If $\tau(v)$ is changed to $\gamma(v)=\tau\left(\left(1+(1 / 2) r_{1}\right) v\right)$, then as before

$$
\operatorname{glb}\{s \in \mathbb{Q}: \gamma \text { makes } w<s v\}=\frac{r_{\beta}}{1+(1 / 2) r_{1}} \leq r_{\beta} .
$$

One also sees that $\tau\left(d w+e v+f v^{\prime}\right)=d \tau(w)+e \tau(v)+f \tau\left(v^{\prime}\right)$ is changed to

$$
d \tau(w)+e\left(1+(1 / 2) r_{1}\right) \tau(v)+f \tau\left(v^{\prime}\right)>d \tau(w)+e \tau(v)+f \tau\left(v^{\prime}\right),
$$

and so

$$
\begin{aligned}
r_{\beta}^{\prime \prime} & =\operatorname{glb}\left\{r \in \mathbb{Q}: \gamma \text { makes } d w+e v+f v^{\prime}<r v^{\prime}\right\} \\
& >\operatorname{glb}\left\{r \in \mathbb{Q}: \tau \text { makes } d w+e v+f v^{\prime}<r v^{\prime}\right\}=r_{\beta}^{\prime}=r_{\beta} .
\end{aligned}
$$

Thus $\gamma$ satisfies $p^{*} \cup\left\{w<r_{\beta}^{\prime \prime} v, d w+e v+f v^{\prime} \mid r_{\beta}^{\prime \prime} v^{\prime}\right\}$ and the argument is complete if $e>0$.

Assume finally that $e<0$. If $r_{\alpha}>0$, then $\tau$ satisfies $p^{*} \cup\left\{(1 / 2) r_{\alpha} v<w\right\}$, where $(1 / 2) r_{\alpha}>0$; so assume that $r_{\alpha} \leq 0$. If $\tau(v)$ is changed to $\gamma(v)=\tau\left(\left(1-(1 / 2) r_{1}\right) v\right)$, then much as before

$$
r_{\alpha}^{\#}=\operatorname{lub}\{r \in \mathbb{Q}: \gamma \text { makes } r v<w\}=\frac{r_{\alpha}}{1-(1 / 2) r_{1}},
$$

which is less than or equal to $r_{\alpha}$ since $0<1-(1 / 2) r_{1}<1$ and $r_{\alpha} \leq 0$. Also,

$$
\begin{aligned}
\gamma\left(d w+e v+f v^{\prime}\right) & =d \tau(w)+e\left(1-(1 / 2) r_{1}\right) \tau(v)+f \tau\left(v^{\prime}\right) \\
& >d \tau(w)+e \tau(v)+f \tau\left(v^{\prime}\right)
\end{aligned}
$$

since $-e(1 / 2) r_{1} \tau(v)>0$. So $\operatorname{lub}\left\{r \in \mathbb{Q}: \gamma\right.$ makes $\left.r v^{\prime}<d w+e v+f v^{\prime}\right\}$ is greater than

$$
\operatorname{lub}\left\{r \in \mathbb{Q}: \tau \text { makes } r v^{\prime}<d w+e v+f v^{\prime}\right\}=r_{\alpha}^{\prime}=r_{\alpha} .
$$

Because $r_{\alpha} \geq r_{\alpha}^{\#}, \gamma$ satisfies $p^{*} \cup\left\{r_{\alpha}^{\#} v \mid w, r_{\alpha}^{\#} v^{\prime}<d w+e v+f v^{\prime}\right\}$ and the argument is complete. 
Lemma 6.24 of [14] provides a $\mathcal{Q}$-formula $\gamma\left(x, x^{\prime}, v, w\right)$ such that for all $a, a^{\prime} \neq 0$ in an e.c. dimension group $\mathscr{D}, \gamma\left(a, a^{\prime}, v, w\right)$ defines in $\mathscr{D}$ the linear function $f: \mathbb{Q} \cdot a \rightarrow \mathbb{Q} \cdot a^{\prime}$ sending $a$ to $a^{\prime}$. So Lemma 3.1 implies the next result.

\section{Corollary $3.2 \quad$ Every f.g. dimension group satisfies}

$$
\begin{aligned}
\forall x & , x^{\prime}>0 \forall y, y^{\prime}\left(x \in \mathbb{Q} \cdot x^{\prime}+\mathbb{Q} \cdot y \vee x^{\prime} \in \mathbb{Q} \cdot x+\mathbb{Q} \cdot y \vee y \in \mathbb{Q} \cdot x+\mathbb{Q} \cdot x^{\prime}\right. \\
& \vee \exists z \in \mathbb{Q} \cdot x(y<z<0) \vee \exists z \in \mathbb{Q} \cdot x(0<z<y) \\
& \vee \exists z \in \mathbb{Q} \cdot x \exists z^{\prime} \in \mathbb{Q} \cdot x^{\prime}\left(\gamma\left(x, x^{\prime}, z, z^{\prime}\right)\right. \\
& \left.\left.\wedge\left[\neg\left(y<z \leftrightarrow y^{\prime}<z^{\prime}\right) \vee \neg\left(z<y \leftrightarrow z^{\prime}<y^{\prime}\right)\right]\right)\right) .
\end{aligned}
$$

One thus reaches the following conclusion.

Theorem 3.3 If $\mathcal{E}$ is a f.g. dimension group, then the automorphisms of $\mathcal{E}$ are multiplications by positive rationals.

Proof One need show merely that any automorphism $\varphi$ of $\mathcal{E}$ is multiplication by a fixed positive rational. Assume that $\varphi$ is not given by such a rational, and fix $v>0$. If $s>0$ in $\mathbb{Q}, \varphi$ is not the automorphism $x \mapsto s x$; so since $\varphi$ is determined by $\varphi(v)$ (see Corollary 2.4), $\varphi(v) \neq s v$. Because $v, \varphi(v)>0, \varphi(v) \notin \mathbb{Q} \cdot v$. Since $\mathcal{E}$ is infinite-dimensional (see [14, Lemma 6.23]), there is $w \in G$ linearly independent of $v, \varphi(v)$. Because $w \notin \mathbb{Q} \cdot v$, there are $\alpha<\beta$ in $\mathbb{R} \backslash \mathbb{Q}$ with

$$
\begin{aligned}
& (t<\alpha \text { iff } t v<w) \quad \text { and } \quad(\alpha<t<\beta \text { iff } t v \mid w) \quad \text { and } \\
& (\beta<t \text { iff } w<t v)
\end{aligned}
$$

for all $t \in \mathbb{Q}$ (see the remarks following [14, Theorem 6.18]). Subtracting a rational multiple of $v$ from $w$ if necessary, one may assume that $\alpha<0<\beta$. If $\left(v^{\prime}, w^{\prime}\right)=(\varphi(v), \varphi(w))$, Corollary 3.2 implies that there is $t \in \mathbb{Q}$ with

$$
\neg\left(w<t v \leftrightarrow w^{\prime}<t v^{\prime}\right) \vee \neg\left(t v<w \leftrightarrow t v^{\prime}<t w^{\prime}\right),
$$

and so $(v, w)$ and $\left(v^{\prime}, w^{\prime}\right)$ have different quantifier-free types. This contradiction yields the result desired.

\section{Automorphisms and Embeddings of i.g. Dimension Groups}

To show that i.g. dimension groups (see [7, Section 5.3]) may have automorphisms that are not multiplications by positive rationals, one may first treat $\omega$-existentially saturated ( $\omega$-e.s.) dimension groups.

Lemma 4.1 In any $\omega$-e.s. dimension group $\mathcal{E}$,

$$
\left\{(\bar{a}, \bar{b}) \in\left(G^{k}\right)^{2}: k \geq 1 \text { and } \bar{a}, \bar{b} \text { have the same quantifier-free } \mathcal{Q} \text {-type in } \mathcal{E}\right\}
$$

is a back-and-forth system.

Proof Because $\mathcal{E}$ is e.c. (see [7, Theorem 5.3.4]), [14, Theorem 3.5] and [14, Corollary 3.6] imply that $k$-tuples from $G$ with the same quantifier-free $\mathcal{Q}$-type have the same existential $\mathcal{Q}$-type. Thus [7, Proof of Theorem 5.3.3(a)] yields the desired result.

One may extend this result to i.g. dimension groups with the help of the following lemma. 
Lemma 4.2 For each positive integer $k$ there is a $\mathcal{Q}$-formula $\varphi_{k}\left(x_{1}, \ldots, x_{k}, y_{1}\right.$, $\left.\ldots, y_{k}\right)$ with the following property: for every e.c. dimension group $\mathcal{D}$ and all $\bar{a}, \bar{b} \in D^{k}$,

$\mathscr{D} \models \varphi_{k}[\bar{a}, \bar{b}] \quad$ iff $\quad \bar{a}$ and $\bar{b}$ have the same quantifier-free Q-type in $\mathscr{D}$.

Proof $\varphi_{1}\left(x_{1}, y_{1}\right)$ may be

$$
\left(x_{1}>0 \leftrightarrow y_{1}>0\right) \wedge\left(x_{1}=0 \leftrightarrow y_{1}=0\right) \wedge\left(x_{1}<0 \leftrightarrow y_{1}<0\right) .
$$

Assume now that $k>1$. With the help of the $\mathcal{Q}$-formula $\gamma(x, y, v, w)$ exploited in the proof of Corollary 3.2, one sees that $\varphi_{k}(\bar{x}, \bar{y})$ may be the conjunction of

$$
\bigwedge_{i=1}^{k}\left(x_{i}=0 \leftrightarrow y_{i}=0\right)
$$

with

$$
\begin{aligned}
& \forall x_{1}^{\prime} \in \mathbb{Q} \cdot x_{1} \cdots \forall y_{k}^{\prime} \in \mathbb{Q} \cdot y_{k}\left[\bigwedge_{i=1}^{k}\left(x_{i} \neq 0 \rightarrow \gamma\left(x_{i}, y_{i}, x_{i}^{\prime}, y_{i}^{\prime}\right)\right)\right. \\
& \left.\quad \rightarrow \varphi_{1}\left(\sum_{i=1}^{k} x_{i}^{\prime}, \sum_{i=1}^{k} y_{i}^{\prime}\right)\right] .
\end{aligned}
$$

One reaches the following conclusion.

Corollary $4.3 \quad$ In any i.g. dimension group $\mathcal{H}$,

$\left\{(\bar{a}, \bar{b}) \in\left(H^{k}\right)^{2}: k \geq 1\right.$ and $\bar{a}, \bar{b}$ have the same quantifier-free $\mathcal{Q}$-type in $\left.\mathscr{H}\right\}$ is a back-and-forth system.

Proof By [7, p. 157] there is an $\omega$-e.s. $\mathscr{E}$ with $\mathscr{H} \preccurlyeq \mathscr{E}$. Lemmas 4.1 and 4.2 imply that $\mathcal{E}$ satisfies

$$
\begin{aligned}
& \left\{\exists x_{1} \exists y_{1} \varphi_{1}\left(x_{1}, y_{1}\right)\right\} \\
& \cup\left\{\forall \bar{x}, x_{k+1}, \bar{y}\left(\varphi_{k}(\bar{x}, \bar{y}) \rightarrow \exists y_{k+1} \varphi_{k+1}\left(\bar{x}, x_{k+1}, \bar{y}, y_{k+1}\right)\right): k \geq 1\right\} \\
& \cup\left\{\forall \bar{x}, \bar{y}, y_{k+1}\left(\varphi_{k}(\bar{x}, \bar{y}) \rightarrow \exists x_{k+1} \varphi_{k+1}\left(\bar{x}, x_{k+1}, \bar{y}, y_{k+1}\right)\right): k \geq 1\right\} .
\end{aligned}
$$

Thus $\mathscr{H} \preccurlyeq \mathscr{G}$ satisfies this set of sentences, and the desired conclusion follows by Lemma 4.2.

So i.g. dimension groups may have more automorphisms than do f.g. dimension groups.

Corollary 4.4 If $\mathscr{H}$ is a countable i.g. dimension group and $a \in H \backslash\{0\}$, then $\mathscr{H}$ has an automorphism $f$ such that $f(a) \neq$ qa for all positive $q \in \mathbb{Q}$.

Proof If one can find $b \in H \backslash\{q a: 0<q \in \mathbb{Q}\}$ with the same quantifier-free 1-type as $a$, Corollary 4.3 and the countability of $\mathscr{H}$ yield an automorphism sending $a$ to $b$. If $a \mid 0$, one may let $b=-a$. If $a>0$, then since $\mathscr{H}$ is directed, it is generated as a group by its positive elements; so since the e.c. dimension group $\mathscr{H}$ is infinite-dimensional (see [14, Lemma 6.23]), there is $b>0$ linearly independent of $a$. A similar argument applies when $a<0$.

The same strategy will produce proper self-embeddings of countable i.g. dimension groups if one can prove the following. 
Theorem 4.5 Let $\mathcal{E}$ be an $\omega$-e.s. dimension group, and let $c$ be an element of $G$; then

$$
\begin{aligned}
& d=\left\{(\bar{a}, \bar{b}) \in\left(G^{k}\right)^{2}: k \geq 1 \text { and } \bar{a}, \bar{b} \text { have the same quantifier-free } \mathbb{Q} \text {-type in } \mathcal{E}\right. \\
& \left.\quad \text { and }\left(\sum_{i=1}^{k} \mathbb{Q} \cdot b_{i}\right) \cap \mathbb{Q} \cdot c=\{0\}\right\}
\end{aligned}
$$

is nonempty and has the following back-and-forth property.

(i) If $(\bar{a}, \bar{b}) \in \mathcal{l}, e \in G$, and $\left(\sum_{i=1}^{k} \mathbb{Q} \cdot b_{i}+\mathbb{Q} \cdot e\right) \cap \mathbb{Q} \cdot c=\{0\}$, then there is $d \in G$ with $((\bar{a}, d),(\bar{b}, e)) \in \mathcal{d}$.

(ii) If $(\bar{a}, \bar{b}) \in \mathcal{d}$ and $d \in G$, then there is $e \in G$ with $((\bar{a}, d),(\bar{b}, e)) \in \mathcal{d}$.

The challenge here is to prove the "forth" direction, and one may begin by describing a special dimension group in which countable dimension groups may be embedded. Let $W$ be the countable set of witnesses (constant symbols) exploited in Sections 2 and 3 , and let $\mathcal{A}$ be the set of closed literals of $\mathcal{Q}_{W}$. Let the $\mathcal{Q}$-structure $\mathscr{D}$ be an $\omega_{1}$-saturated divisible ordered abelian group.

Lemma 4.6 Let $S \subseteq \mathcal{A}$ and $S_{+} \subseteq S$ consist of the atomic sentences in $S$. For each $\varphi \in S \backslash S_{+}$, let $S_{\varphi}=S_{+} \cup\{\varphi\}$.

(i) If $S=S_{+}$, then $S$ is satisfiable in some dimension group just in case $S$ is satisfiable in $D$.

(ii) If $S \neq S_{+}$, then $S$ is satisfiable in some dimension group just in case every $S_{\varphi}$ may be satisfied in $\mathscr{D}$; in this case $S$ may be satisfied in $\mathscr{D}^{\left|S \backslash S_{+}\right|}$.

(iii) $S$ is satisfiable in some dimension group if and only if $S$ is satisfiable in $\mathscr{D}^{\omega}$.

Proof Condition (iii) clearly follows from (i) and (ii). Since every nontrivial power of $\mathscr{D}$ is a dimension group, one may reach the desired conclusion by going from left to right in (i) and (ii) and by showing that $S$ is satisfiable in $\mathscr{D}^{\left|S \backslash S_{+}\right|}$if each $S_{\varphi}$ is satisfiable in $\mathcal{D}$.

Suppose that $S=S_{+}$is satisfied in the (divisible) dimension group $\mathcal{M}$. If $M=\{0\}$, there is nothing to prove; so assume that $M \neq\{0\}$. As in [14, Section 2], $\mathcal{M}$ may be embedded by the diagonal map in the direct product

$$
\widehat{\mathcal{M}}=\prod_{\prec \in \mathcal{O}(\mathcal{M})}(M, \prec),
$$

where $\mathcal{O}(\mathcal{M})$ is the set of all linear orderings $\prec$ of $M$ that extend $\mathcal{M}$ 's partial ordering and make $(M, \prec)$ a (divisible) ordered abelian group. So the at most countable set $S=S_{+}$may be satisfied in a countable elementary submodel of any $(M, \prec)$, and so in the $\omega_{1}$-saturated $\mathscr{D}$.

Suppose now that $S \neq S_{+}$is satisfied in the (divisible) dimension group $\mathcal{M}$. As above, one may assume that $M \neq\{0\}$ and that $\mathcal{M}$ is embedded in $\widehat{\mathcal{M}}$ by the diagonal map; so $S$ is satisfied in $\widehat{\mathcal{M}}$. If $\varphi \in S \backslash S_{+}$, then the negated-atomic sentence $\varphi$ is satisfied in some factor $(M, \prec)$ of $\widehat{\mathcal{M}}$, as are the atomic sentences in $S_{+}$; so $S_{\varphi}$ is satisfied in $(M, \prec)$, and the earlier argument shows that $S_{\varphi}$ is satisfied in $\mathscr{D}$.

Assume now that $S \neq S_{+}$and that every $S_{\varphi}$ may be satisfied in $\mathscr{D}$. If the assignment $\rho_{\varphi}$ makes $S_{\varphi}$ true in $\mathscr{D}$ for every $\varphi \in S \backslash S_{+}$, then $S$ may be satisfied by the 
assignment $\rho$ in $\mathscr{D}^{\left|S \backslash S_{+}\right|}$with

$$
\rho(w)=\left(\rho_{\varphi}(w)\right)_{\varphi \in S \backslash S_{+}}
$$

for every witness $w$.

Now one may prove the "forth" direction in Theorem 4.5.

Lemma 4.7 Let $\mathcal{E}$ be an $\omega$-e.s. dimension group, and let $c, d \in G, k \geq 0$, and $\bar{a}, \bar{b} \in G^{k}$. If $\bar{a}$ and $\bar{b}$ have the same quantifier-free $\mathbb{Q}$-type in $\mathcal{G}$ and $\left(\sum_{i=1}^{k} \mathbb{Q} \cdot b_{i}\right) \cap \mathbb{Q} \cdot c=\{0\}$, then there is $e \in G$ such that $\left(\sum_{i=1}^{k} \mathbb{Q} \cdot b_{i}+\mathbb{Q} \cdot e\right) \cap$ $\mathbb{Q} \cdot c=\{0\}$ and $(\bar{a}, d)$ and $(\bar{b}, e)$ have the same quantifier-free $\mathbb{Q}$-type in $\mathcal{E}$.

Proof The argument will go by induction on $k \geq 0$.

Suppose that $k=0$. If $d=0$, one may let $e=0$; so assume that $d \neq 0$. Because $\mathcal{G}$ is directed, it is generated by its positive elements; because $\mathcal{E}$ is e.c., it is generated by the elements incomparable with 0 (see [14, Lemma 6.9]). So since the e.c. dimension group $\mathcal{E}$ is infinite-dimensional (see [14, Lemma 6.23]), the desired conclusion follows.

Assume now that $k>0$ and that tuples of length less than $k$ obey the result. If $d \in \sum_{i=1}^{k} \mathbb{Q} \cdot a_{i}$, the desired result follows because $\bar{a}$ and $\bar{b}$ have the same quantifierfree type and $\left(\sum_{i=1}^{k} \mathbb{Q} \cdot b_{i}\right) \cap \mathbb{Q} \cdot c=\{0\}$; so assume that $d \notin \sum_{i=1}^{k} \mathbb{Q} \cdot a_{i}$. If the quantifier-free type of $(\bar{a}, d)$ contains a nontrivial identity, then $\bar{a}$ must be linearly dependent, and the desired conclusion follows by induction hypothesis; so assume that the quantifier-free type of $(\bar{a}, d)$ contains no nontrivial identities. Because $\mathcal{E}$ is $\omega$-e.s., one may finish the proof by showing the following: if $\pi_{1}(\bar{x}, y), \ldots, \pi_{l}(\bar{x}, y)$ are atomic $\mathcal{Q}$-formulas with

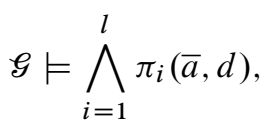

$\eta_{1}(\bar{x}, y), \ldots, \eta_{m}(\bar{x}, y)$ are negated-atomic $Q$-formulas with

$$
\mathcal{E} \models \bigwedge_{j=1}^{m} \eta_{j}(\bar{a}, d),
$$

and $f_{1}(\bar{x}, y, z), \ldots, f_{n}(\bar{x}, y, z)$ are $\mathbb{Q}$-linear forms in which $y$ occurs nontrivially, then there is $e \in G$ with

$$
\mathcal{E} \models \bigwedge_{i=1}^{l} \pi_{i}(\bar{b}, e) \wedge \bigwedge_{j=1}^{m} \eta_{j}(\bar{b}, e) \wedge \bigwedge_{s=1}^{n} f_{s}(\bar{b}, e, c) \neq 0 .
$$

Since $\bar{a}$ and $\bar{b}$ have the same quantifier-free type and $\mathcal{G}$ is e.c., [14, Theorem 3.5] and [14, Corollary 3.6] provide $e_{0} \in G$ with

$$
\mathcal{E} \models \bigwedge_{i=1}^{l} \pi_{i}\left(\bar{b}, e_{0}\right) \wedge \bigwedge_{j=1}^{m} \eta_{j}\left(\bar{b}, e_{0}\right) .
$$

$H=\sum_{i=1}^{k} \mathbb{Q} \cdot b_{i}+\mathbb{Q} \cdot c+\mathbb{Q} \cdot e_{0}$ is the domain of a substructure $\mathcal{H}$ of $\mathcal{E}$, and Lemma 4.6 implies that the quantifier-free type of $\left(\bar{b}, c, e_{0}\right)$ may be realized in $\mathscr{D}^{\omega}$; so there is a $\mathcal{Q}$-embedding $g: \mathscr{H} \rightarrow \mathscr{D}^{\omega}$. Because $\mathscr{D}^{\omega}$ is the direct product of countably many copies of $\mathscr{D}$, one may assume that each negated-atomic sentence 
$\eta_{j}\left(g(\bar{b}), g\left(e_{0}\right)\right)$ is true in infinitely many coordinates of $\mathbb{D}^{\omega}$. So there are distinct coordinates $k_{1}, \ldots, k_{m}$ such that

$$
\mathscr{D} \models \bigwedge_{j=1}^{m} \eta_{j}\left(g(\bar{b})_{k_{j}},\left(g\left(e_{0}\right)\right)_{k_{j}}\right) .
$$

Since the quantifier-free type of $(\bar{a}, d)$ contains no nontrivial identities, one may assume that each $\pi_{i}$ is a (strict) inequality. So for $j=1, \ldots, m$ there is an interval $\left(p_{j}, q_{j}\right) \ni g\left(e_{0}\right)_{k_{j}}$ on which every $\pi_{i}\left(g(\bar{b})_{k_{j}}, y\right)$ is true. For those $\eta_{j}$ 's that are negated identities, one may assume that $\eta_{j}\left(g(\bar{b})_{k_{j}}, y\right)$ is true on $I_{j}=\left(p_{j}, q_{j}\right)$; for those $\eta_{j}$ 's that are negated (linear) inequalities, one may assume that $\eta_{j}\left(g(\bar{b})_{k_{j}}, y\right)$ is true on $I_{j} \subseteq\left(p_{j}, q_{j}\right)$, where $I_{j}$ is $\left.\left(p_{j}, g\left(e_{0}\right)\right)_{k_{j}}\right]$ or $\left.\left[g\left(e_{0}\right)\right)_{k_{j}}, q_{j}\right)$. Because each $I_{j}$ is infinite and $y$ occurs nontrivially in each linear polynomial $f_{s}\left(g(\bar{b})_{k_{j}}, y, g(c)_{k_{j}}\right)$, there is $r_{j} \in I_{j}$ with

$$
\mathscr{D} \models \bigwedge_{s=1}^{n} f_{s}\left(g(\bar{b})_{k_{j}}, r_{j}, g(c)_{k_{j}}\right) \neq 0 .
$$

Let $e_{1} \in \mathscr{D}^{\omega}$ result from $g\left(e_{0}\right)$ when for $j=1, \ldots, m, g\left(e_{0}\right)_{k_{j}}$ is changed to $r_{j}$; then

$$
\mathscr{D}^{\omega} \models \bigwedge_{i=1}^{l} \pi_{i}\left(g(\bar{b}), e_{1}\right) \wedge \bigwedge_{j=1}^{m} \eta_{j}\left(g(\bar{b}), e_{1}\right) \wedge \bigwedge_{s=1}^{n} f_{s}\left(g(\bar{b}), e_{1}, g(c)\right) \neq 0 .
$$

The amalgamation property for dimension groups (see [14, Theorem 4.1]), together with remarks at the end of [14, Section 4], provides a divisible dimension group $\mathcal{K}$ and $\mathcal{Q}$-embeddings $h: \mathscr{D}^{\omega} \rightarrow \mathcal{K}$ and $r: \mathscr{E} \rightarrow \mathcal{K}$ such that

$$
h \circ g=r \uparrow H \text {. }
$$

So $\mathcal{K}$ satisfies

$$
\begin{gathered}
\bigwedge_{i=1}^{l} \pi_{i}\left((h \circ g)(\bar{b}), h\left(e_{1}\right)\right) \wedge \bigwedge_{j=1}^{m} \eta_{j}\left((h \circ g)(\bar{b}), h\left(e_{1}\right)\right) \\
\quad \wedge \bigwedge_{s=1}^{n} f_{s}\left((h \circ g)(\bar{b}), h\left(e_{1}\right),(h \circ g)(c)\right) \neq 0,
\end{gathered}
$$

and by the next-to-last display

$$
\mathcal{K} \models \exists y\left(\bigwedge_{i=1}^{l} \pi_{i}(r(\bar{b}), y) \wedge \bigwedge_{j=1}^{m} \eta_{j}(r(\bar{b}), y) \wedge \bigwedge_{s=1}^{n} f_{s}(r(\bar{b}), y, r(c)) \neq 0\right) .
$$

Because $\mathcal{E}$ is e.c. and $r: \mathcal{E} \rightarrow \mathcal{K}$ is an embedding,

$$
\mathcal{E} \models \exists y\left(\bigwedge_{i=1}^{l} \pi_{i}(\bar{b}, y) \wedge \bigwedge_{j=1}^{m} \eta_{j}(\bar{b}, y) \wedge \bigwedge_{s=1}^{n} f_{s}(\bar{b}, y, c) \neq 0\right),
$$

as desired.

Lemma 4.7 implies that $d$ is nonempty and obeys the "forth" direction, while Lemma 4.1 gives the "back" direction; so Theorem 4.5 is established.

It applies also to i.g. dimension groups. 
Corollary 4.8 If $\mathscr{H}$ is an i.g. dimension group and $c \in H$, then

$$
\begin{aligned}
\mathscr{l}= & \left\{(\bar{a}, \bar{b}) \in\left(H^{k}\right)^{2}: k \geq 1 \text { and } \bar{a}, \bar{b} \text { have the same quantifier-free } \mathbb{Q} \text {-type in } \mathcal{H}\right. \\
& \text { and } \left.\left(\sum_{i=1}^{k} \mathbb{Q} \cdot b_{i}\right) \cap \mathbb{Q} \cdot c=\{0\}\right\}
\end{aligned}
$$

is nonempty and has the back-and-forth property.

Proof Note that by [14, Lemma 6.21] there is for each positive integer $k$ a $Q$-formula $\lambda_{k}\left(x_{1}, \ldots, x_{k}, z\right)$ with the following property: if $\mathcal{G}$ is any e.c. dimension group and $e_{1}, \ldots, e_{k}, f \in G$, then $\mathcal{E} \models \lambda_{k}(\bar{e}, f)$ just in case $\left(\sum_{i=1}^{k} \mathbb{Q} \cdot e_{i}\right) \cap \mathbb{Q} \cdot f=\{0\}$. So Corollary 4.8 follows from Theorem 4.5 much as Corollary 4.3 follows from Lemma 4.1 .

One may exploit this result to learn more about automorphisms.

Corollary 4.9 If $\mathscr{H}$ is a countable i.g. dimension group and $c \in H$, then there is

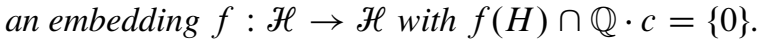

Proof Given $d$ as in Corollary 4.8, one may exploit the countability of $\mathscr{H}$ as in the usual back-and-forth arguments to produce $f$.

\section{Recursion-Theoretic Complexity of the Finite-Forcing Companion}

One may start with a fact about e.c. dimension groups.

Lemma 5.1 There is an $\exists \forall \exists$-formula $\pi(x, y, z, w)$ such that if $a \neq 0$ in the e.c. dimension group $\mathcal{E}$, then $\pi(a, y, z, w)$ defines $\{(p a, q a,(p q) a): p, q \in \mathbb{Q}\}$ in $\mathscr{E}$.

Proof As noted in Section 3, [14, Lemma 6.24] provides an $\exists \forall \exists$-formula $\gamma(x, y, v, w)$ such that for all nonzero elements $a, a^{\prime}$ of any e.c. dimension group $\mathcal{G}$, $\gamma\left(a, a^{\prime}, v, w\right)$ defines in $\mathcal{G}$ the linear function $g: \mathbb{Q} \cdot a \rightarrow \mathbb{Q} \cdot a^{\prime}$ sending $a$ to $a^{\prime}$. Let $\pi(x, y, z, w)$ be the formula

$$
y, z, w \in \mathbb{Q} \cdot x \wedge(y=0 \vee z=0 \rightarrow w=0) \wedge(y, z \neq 0 \rightarrow \gamma(x, y, z, w)) .
$$

Since $y \in \mathbb{Q} \cdot x$ is $\exists \forall, \pi(x, y, z, w)$ is $\exists \forall \exists$.

Suppose that $p, q \in \mathbb{Q}$. If $p$ or $q$ is $0,(p q) a=0$ and $\pi(a, p a, q a,(p q) a)$; so assume that $p, q \neq 0$. Then $p a, q a \neq 0$ in $\mathcal{E}$, and if $g: \mathbb{Q} \cdot a \rightarrow \mathbb{Q} \cdot p a$ is the linear function sending $a$ to $p a$, then $g(q a)=(p q) a$ and $\pi(a, p a, q a,(p q) a)$.

Suppose conversely that $\pi(a, b, c, d)$ in $\mathcal{E}$. Because $b, c$, and $d$ belong to $\mathbb{Q} \cdot a$, there are rationals $p, q$, and $r$ with $b=p a, c=q a$, and $d=r a$. If $b$ or $c$ is 0 , then $p$ or $q$ is $0, p q$ is 0 , and $d=0=(p q) a$. If neither $b$ nor $c$ is 0 , and $g: \mathbb{Q} \cdot a \rightarrow \mathbb{Q} \cdot b=\mathbb{Q} \cdot p a$ is the linear function sending $a$ to $b=p a$, then $d=g(q a)=(p q) a$. So $(a, b, c, d)=(a, p a, q a,(p q) a)$, and the argument is complete.

If $\psi(\bar{y})$ is an unnested formula (see [8, p. 58]), in the language of rings, that does not contain the variable $x$, let $\psi^{(x)}(\bar{y})$ result from $\psi$ when all quantifiers are relativized to $\mathbb{Q} \cdot x$, all occurrences of 1 are replaced by $x$, and all subformulas $r \cdot s=t$ are replaced by $\pi(x, r, s, t)$. Lemma 5.1 implies that the $\mathcal{Q}$-formula $\psi^{(x)}(\bar{y})$ has a special property. 
Corollary 5.2 If $a \neq 0$ in the e.c. dimension group $\mathcal{E}$ and $q_{1}, \ldots, q_{k} \in \mathbb{Q}$, then

$$
(\mathbb{Q},+,-, \cdot, 0,1) \models \psi\left(q_{1}, \ldots, q_{k}\right)
$$

if and only if

$$
\mathcal{E} \models \psi^{(a)}\left(q_{1} a, \ldots, q_{k} a\right) .
$$

One simple corollary runs as follows.

Theorem 5.3 The theory of the ring of rational numbers is one-one reducible to the $Q$-theory of e.c. dimension groups.

Proof If $\varphi$ is an unnested sentence, not containing $x$, in the language of rings, then $(\mathbb{Q},+,-, \cdot, 0,1) \models \varphi$ just in case $\forall x\left(x \neq 0 \rightarrow \varphi^{(x)}\right)$ is true in every e.c. dimension group.

One reaches a stronger conclusion for f.g. dimension groups.

Theorem 5.4 The theory of the ring of rational numbers is recursively isomorphic to the finite-forcing companion $T^{\mathrm{f}}$ of the $\mathbb{Q}$-theory of dimension groups.

Proof Two sets are recursively isomorphic just in case each is one-one reducible to the other. Corollary 5.2 again implies that if $\varphi$ is an unnested sentence, not containing $x$, in the language of rings, then $(\mathbb{Q},+,-, \cdot, 0,1) \models \varphi$ just in case $\forall x\left(x \neq 0 \rightarrow \varphi^{(x)}\right)$ is true in every f.g. dimension group. Conversely, $T^{\mathrm{f}}$ is one-one reducible to true arithmetic (see [6, Theorem 7.7]) ${ }^{6}$ and so to the theory of the ring of rationals, in which $\mathbb{Z}$ is definable (see Robinson [10, Theorem 3.1]).

By contrast, the finite-forcing companion of the $\{+,-, \wedge, \vee, 0\}$-theory of abelian $l$-groups is recursive (by Weispfenning [15, Theorem 3.6.18] and the fact that all f.g. abelian $l$-groups are elementarily equivalent). Yet the finite-forcing companion of the $\{+,-, \wedge, \vee, 0\}$-theory of $l$-groups is recursively isomorphic to first-order arithmetic (by Glass and Pierce [5, proof of Theorem 18]).

Call $\gamma \in \mathbb{R}$ representable just in case $(-\infty, \gamma) \cap \mathbb{Q}$ is definable in the ring $\mathbb{Q}$. With the help of [10], one sees that $\gamma$ is representable just in case $\left\{2^{m} 3^{n}: m, n \in \mathbb{N}, m /(m+1)<|\gamma|\right\}$ is arithmetic: that is, definable in $(\mathbb{N},+, \cdot, 0,1)$. So the representable reals include all the recursive reals, and in particular all algebraic reals as well as familiar transcendental numbers like $e$ and $\pi$. Yet Lemma 2.1 allows one to show the following.

Theorem 5.5 Let $\mathcal{E}$ be an f.g. dimension group containing elements $a>0$ and $b \notin \mathbb{Q} \cdot a$. If $\alpha, \beta \in \mathbb{R} \backslash \mathbb{Q}$ obey

$$
\begin{aligned}
& (t<\alpha \text { iff } t a<b) \quad \text { and } \quad(\alpha<t<\beta \text { iff } t a \mid b) \quad \text { and } \\
& (\beta<t \text { iff } b<t a)
\end{aligned}
$$

for all $t \in \mathbb{Q}$, then neither $\alpha$ nor $\beta$ is representable.

Proof The argument below will handle $\alpha$. If $\gamma \in \mathbb{R}$ is representable, then there is an unnested formula $\Gamma(y)$, in the language of rings, that defines $(-\infty, \gamma) \cap \mathbb{Q}$ and does not contain the variable $x$. Lemma 2.1, applied to the function $f$ projecting on the first coordinate, implies that in any finitely generic dimension group,

$$
\begin{aligned}
& \forall x>0 \forall y\left(y \notin \mathbb { Q } \cdot x \rightarrow \exists z \in \mathbb { Q } \cdot x \left(\left(\Gamma^{(x)}(z) \wedge z \mid y\right)\right.\right. \\
& \left.\left.\quad \vee\left(\forall w\left(\Gamma^{(x)}(w) \rightarrow w<z\right) \wedge z<y\right)\right)\right) .
\end{aligned}
$$


So there is $k \in \mathbb{Q}$ such that either $k<\gamma$ and $k a \mid b$ or $\gamma \leq k$ and $k a<b$. If $k<\gamma$ and $k a \mid b$, then $\alpha<k<\gamma$; if $\gamma \leq k$ and $k a<b$, then $\gamma \leq k<\alpha$. In either case $\alpha \neq \gamma$.

So one reaches the following conclusion.

Corollary 5.6 Nof.g. dimension group is arithmetic.

Proof Suppose that the f.g. dimension group $\mathcal{E}$ has an arithmetic diagram. $\mathcal{E}$ contains elements $a>0$ and $b \notin \mathbb{Q} \cdot a$, and since $\mathcal{E}$ 's diagram is arithmetic, $\{r \in \mathbb{Q}: r a<b\}$ is definable in the ring $\mathbb{Q}$. Thus the $\alpha$ corresponding to $(a, b)$ as in Theorem 5.5 is representable, contrary to Theorem 5.5.

By contrast, the abelian $l$-group $\ell$ that is shown to be f.g. in [12, Section 1] certainly is recursive.

The following definition allows for further applications of Lemma 2.1. Call the continuous function $f: \mathbb{R}^{2} \rightarrow \mathbb{R}$ representable just in case $\left\{(r, s, t) \in \mathbb{Q}^{3}: t<\right.$ $f(r, s)\}$ is definable in the ring $\mathbb{Q}$.

Theorem 5.7 Let $f: \mathbb{R}^{2} \rightarrow \mathbb{R}$ be continuous, strictly increasing in both arguments, and representable, and let $\gamma \in \mathbb{R}$ be representable. If $a, b, \alpha$, and $\beta$ are as in Theorem 5.5, then $f(\alpha, \beta) \neq \gamma$.

Proof Let $\Phi(y, z, w)$ be an unnested formula, in the language of rings, defining $\left\{(r, s, t) \in \mathbb{Q}^{3}: t<f(r, s)\right\}$ in the ring $\mathbb{Q}$, and let $\Gamma(y)$ be an unnested formula, in the language of rings, defining $(-\infty, \gamma) \cap \mathbb{Q}$ in the ring $\mathbb{Q}$. Without loss of generality, neither $\Phi$ nor $\Gamma$ contains the variable $x$. Lemma 2.1 implies that every f.g. dimension group obeys

$$
\begin{aligned}
& \forall x>0 \forall y(y \notin \mathbb{Q} \cdot x \rightarrow \exists k, l, m \in \mathbb{Q} \cdot x \\
& \quad\left[\left(\Gamma^{(x)}(m) \wedge \forall t\left(\Phi^{(x)}(k, l, t) \rightarrow t<m\right) \wedge k \mid y \wedge y<l\right)\right. \\
& \left.\left.\quad \vee\left(\Phi^{(x)}(k, l, m) \wedge \forall t\left(\Gamma^{(x)}(t) \rightarrow t<m\right) \wedge k<y \wedge l \mid y\right)\right]\right) .
\end{aligned}
$$

So there are $k, l, m \in \mathbb{Q}$ such that either

$$
m<\gamma, \quad f(k, l) \leq m, \quad k a \mid b, \quad \text { and } \quad b<l a
$$

or

$$
m<f(k, l), \quad \gamma \leq m, \quad k a<b, \quad \text { and } \quad l a \mid b .
$$

In the first case $\alpha<k<\beta<l$, and so $f(\alpha, \beta)<f(k, l) \leq m<\gamma$ since $f$ is strictly increasing in both arguments. In the second case $k<\alpha<l<\beta$, and so $f(k, l)<f(\alpha, \beta)$ since $f$ is strictly increasing in both arguments and $\gamma \leq m<f(k, l)<f(\alpha, \beta)$. In either case $f(\alpha, \beta) \neq \gamma$.

So, for example, $e^{\alpha}+\beta+\cos \beta \neq \sqrt{\pi}$. Lemma 2.1 may be generalized to handle continuous $f: \mathbb{R}^{2} \rightarrow \mathbb{R}$ strictly monotone in one argument, and Theorem 5.7 may be generalized to handle continuous, representable $f: \mathbb{R}^{2} \rightarrow \mathbb{R}$ strictly monotone in both arguments. 


\section{Recursion-Theoretic Complexity of the Infinite-Forcing Companion}

As defined in [7, Section 5.3], the infinite-forcing companion $T^{\mathrm{F}}$ of the theory $T^{\mathcal{Q}}$ of divisible dimension groups is the theory of the class of all i.g. dimension groups. Though $[6$, p. 70$]$ defines the infinite-forcing companion in the same way from the class of infinite-generic structures, the definition of infinite-generic in [6, pp. 55-56] is very different from the definition in [7, Section 5.3]. But the two definitions yield the same class of structures by [6, Theorem 3.10] and [7, Exercise 5.3.7]; so the results in [6] about the infinite-forcing companion apply to $T^{\mathrm{F}}$ and yield the following conclusion.

Lemma 6.1 $\quad T^{\mathrm{F}}$ is one-one reducible to second-order arithmetic.

Proof This result is [6, Theorem 7.6].

To show that second-order arithmetic is one-one reducible to $T^{\mathrm{F}}$-and that the two theories are therefore recursively isomorphic_one may translate the language of second-order arithmetic into $\mathcal{Q}$ in such a way that a sentence of second-order arithmetic is true just in case its translation is true in all i.g. dimension groups. Because $T^{\mathcal{Q}}$ has the amalgamation property (see [14, Theorem 4.1]) and $\{0\}$ embeds into every model of $T^{\mathcal{Q}}$, it has the joint embedding property. Thus $T^{\mathrm{F}}$ is complete (see [7, p. 157]) and is the theory of any particular i.g. dimension group. The results of Section 5 show how from $a>0$ a copy $\mathbb{Q} \cdot a$ of the ring of rationals may be defined in any e.c. dimension group $\mathcal{E}$ containing $a$; and if $\mathcal{E}$ is $\omega$-e.s., every real number $r$ corresponds to many elements of $\mathcal{E}$ that make in $\mathbb{Q} \cdot a$ the cut $r$ makes in $\mathbb{Q}$. By encoding sets of natural numbers by special irrational numbers one may hope to interpret second-order arithmetic in $\mathscr{G}$. Because $\omega$-e.s. dimension groups are i.g., such an interpretation would yield the desired conclusion about $T^{\mathrm{F}}$. In the following sketch of an interpretation, $A \subseteq \mathbb{N}$ will correspond to the real number

$$
\sum_{i=0}^{\infty} \chi_{A}(i) 10^{-(i+1)^{2}}
$$

where

$$
\chi_{A}(i)= \begin{cases}2 & \text { if } i \in A \\ 1 & \text { if } i \notin A\end{cases}
$$

for all $i \in \mathbb{N}$. by

Let $S=\{1,2\}^{\mathbb{N}}$, let $\sqsubset$ be the lexicographic order on $S$, and define $N: S \rightarrow \mathbb{R}$

$$
N(a)=\sum_{i=0}^{\infty} a(i) 10^{-(i+1)^{2}} .
$$

One may easily show the following.

Lemma 6.2 If $a \sqsubset b$, then $N(a)<N(b)$.

Proof Suppose that $a(i)<b(i)$ while $a(j)=b(j)$ for all $j<i$. If

$$
p=\sum_{j<i} a(j) 10^{-(j+1)^{2}}=\sum_{j<i} b(j) 10^{-(j+1)^{2}},
$$


then

$$
\begin{aligned}
N(a) & =p+10^{-(i+1)^{2}}+\sum_{l>i} a(l) 10^{-(l+1)^{2}} \\
& \leq p+10^{-(i+1)^{2}}+\sum_{l \geq(i+1)^{2}+1} 2 \cdot 10^{-l} \\
& =p+\frac{11}{9} 10^{-(i+1)^{2}}
\end{aligned}
$$

while

$$
\begin{aligned}
N(b) & =p+2 \cdot 10^{-(i+1)^{2}}+\sum_{l>i} b(l) 10^{-(l+1)^{2}} \\
& >p+2 \cdot 10^{-(i+1)^{2}} \\
& >p+\frac{11}{9} \cdot 10^{-(i+1)^{2}} \geq N(a) .
\end{aligned}
$$

So if $\mathcal{N}=\operatorname{ran}(N) \subseteq(0,1)$, then $N$ is an order-isomorphism of $(S, \sqsubset)$ onto $(\mathcal{N},<)$. Let $S^{\prime}$ be the set of finite nonempty sequences from $\{1,2\}$, and for $c \in S^{\prime}$ let $\operatorname{lh}(c)$ be the length ${ }^{7}$ of $c$ and

$$
N^{\prime}(c)=\sum_{i<\operatorname{lh}(c)} c(i) 10^{-(i+1)^{2}} .
$$

So $\mathcal{N}^{\prime}=\operatorname{ran}\left(N^{\prime}\right)$ is a set of rational numbers with finite decimal expansions. If $a \in S, N(a)$ is the least upper bound of $\left\{N^{\prime}(a \uparrow n)\right\}_{0<n \in \mathbb{N}}$, a strictly increasing sequence of rational numbers. The proof of Lemma 6.1 shows that if $a \in S, c \in S^{\prime}$, and $a \sqsubset(c, \overrightarrow{1})$, then $N(a)<N^{\prime}(c)$. So if $a \in S$ and $i \in \mathbb{N}$, digit number $(i+1)^{2}$ in the decimal expansion of $N(a)$ is the same as digit number $(i+1)^{2}$ in the decimal expansion of the largest element of

$$
\left\{r \in \mathcal{N}^{\prime}: r<N(a) \text { and } r=N^{\prime}(c) \text { for some } c \text { of length } i+1\right\},
$$

a finite subset of $\mathcal{N}^{\prime}$. These observations are behind the translation, described below, of the language of second-order arithmetic into $\mathcal{Q}$.

Consider the following primitive-recursive functions:

$$
\begin{aligned}
f: a \in \mathbb{N} & \mapsto \begin{cases}1 \quad & \text { if } a=0, \\
2 & \text { if } a \neq 0,\end{cases} \\
h: a \in \mathbb{N} \mapsto & 10^{\left(a^{2}\right)}, \\
k:(a, i) \in \mathbb{N}^{2} \mapsto & \sum_{j<i} f\left((a)_{j}\right) 10^{i^{2}-(j+1)^{2}} \\
& \left((a)_{j} \text { is the power to which the } j \text { th prime } p_{j} \text { divides } a\right) .
\end{aligned}
$$

By the discussion in Section 5 there are $\mathcal{Q}$-formulas $\Phi(x, y, z), H(x, y, z)$, and $K(x, y, z, w)$ such that if $\mathcal{E}$ is an e.c. dimension group and $b \in G \backslash\{0\}$, then $\Phi(b, y, z), H(b, y, z)$, and $K(b, y, z, w)$ define in $\mathcal{G}$ the functions $f_{b}: \mathbb{N} \cdot b \rightarrow \mathbb{N} \cdot b$, $h_{b}: \mathbb{N} \cdot b \rightarrow \mathbb{N} \cdot b$, and $k_{b}:(\mathbb{N} \cdot b)^{2} \rightarrow \mathbb{N} \cdot b$ corresponding to $f, h$, and $k$ under the bijection $I_{b}: \mathbb{N} \rightarrow \mathbb{N} \cdot b$ that sends each $n \in \mathbb{N}$ to $n b$. Section 5 also provides a $\mathbb{Q}$-formula-written " $y \in \mathbb{N} \cdot x$ " in what follows-such that $y \in \mathbb{N} \cdot a$ defines $\mathbb{N} \cdot a$ in any e.c. dimension group containing $a$. With the help of the formula 
$\pi(x, y, z, w)$ defining rational multiplication (see Lemma 5.1), one may build the Q-formula $\lambda(x, y, z)=$

$$
\begin{aligned}
& x, y \neq 0 \wedge y \in \mathbb{N} \cdot x \wedge z \in \mathbb{Q} \cdot x \wedge \exists w \in \mathbb{N} \cdot x \\
& \quad\left(w \neq 0 \wedge \exists w^{\prime}, w^{\prime \prime}\left(H\left(x, y, w^{\prime}\right) \wedge K\left(x, w, y, w^{\prime \prime}\right) \wedge \pi\left(x, w^{\prime}, z, w^{\prime \prime}\right)\right)\right),
\end{aligned}
$$

which has the following property: if $\mathcal{E}$ is an e.c. dimension group, $b \in G \backslash\{0\}$, and $0<l \in \mathbb{N}$, then $\lambda(b, l b, z)$ defines

$$
\left\{r b: r \in \mathcal{N}^{\prime} \text { and } r=N^{\prime}(c) \text { for some } c \text { of length } l\right\}
$$

in $\mathcal{G}$. So if $v(x, z)$ is $\exists y \lambda(x, y, z), v(b, z)$ defines $\mathcal{N}^{\prime} b$ whenever $b \neq 0$ in the e.c. dimension group $\mathcal{E}$. Let $\mathcal{l}(x, y)$ be the $\mathcal{Q}$-formula

$$
\begin{aligned}
x> & 0 \wedge \exists z, w \in \mathbb{Q} \cdot x(z<y<w) \\
& \wedge \forall z \in \mathbb{Q} \cdot x[(z<y \vee y<z) \wedge(z<y \rightarrow \exists w \in \mathbb{Q} \cdot x(z<w<y)) \\
& \wedge(y<z \rightarrow \exists w \in \mathbb{Q} \cdot x(y<w<z))] ;
\end{aligned}
$$

then if $\mathcal{G}$ is any e.c. dimension group and $0<b$ in $\mathcal{G}, \mathcal{E} \models d(b, c)$ just in case

$$
(\{r \in \mathbb{Q}: r b<c\},\{r \in \mathbb{Q}: r b>c\}) \quad(=P(b, c))
$$

is the cut made in $\mathbb{Q}$ by an irrational number. The $\mathcal{Q}$-formula $\mathscr{f}(x, y)=$

$$
\mathcal{l}(x, y) \wedge \forall z \in \mathbb{Q} \cdot x\left(z<y \rightarrow \exists z^{\prime}\left(v\left(x, z^{\prime}\right) \wedge z<z^{\prime}<y\right)\right)
$$

has the following property: if $\mathcal{E}$ is an e.c. dimension group, $0<b \in G$, and $c \in G$, $\mathcal{E} \models \mathcal{L}(b, c)$ just in case $P(b, c)$ is the cut in $\mathbb{Q}$ made by some element of $\mathcal{N}$. Finally, there is a $\mathcal{Q}$-formula $\mu(x, y, z)$ with the following property: if $\mathcal{G}$ is an e.c. dimension group, $b, c, d \in G$, and $0<b$, then $\mathcal{E} \models \mu(b, c, d)$ just in case $P(b, c)$ is the cut made in $\mathbb{Q}$ by $N(a)$ for some $a \in S$, and $d=k b$, where $k \in \mathbb{N}$ and 2 is digit number $(k+1)^{2}$ in the decimal expansion of $N(a)$. To find digit number $(k+1)^{2}$ in $N(a)$ 's decimal expansion, look for the greatest $N^{\prime}(c)<N(a)$ with $c$ of length $k+1$. Digit number $(k+1)^{2}$ in the decimal expansion of $N(a)$ is the same as digit number $(k+1)^{2}$ in the decimal expansion of $N^{\prime}(c)$. If $k=0$, multiply $N^{\prime}(c)$ by 10 to get the digit. If $k>0$, find the greatest $N^{\prime}(d)<N(a)$ with $d$ of length $k$; then $N^{\prime}(c)-N^{\prime}(d)$ will have just one decimal digit-the one of interest here-and it will equal $10^{(k+1)^{2}}\left(N^{\prime}(c)-N^{\prime}(d)\right)$. With this procedure in mind, one sees that $\mu(x, y, z)$ may be the $\mathcal{Q}$-formula

$$
\begin{aligned}
x> & 0 \wedge \mathcal{g}(x, y) \wedge z \in \mathbb{N} \cdot x \\
& \wedge \exists w\left(\lambda(x, z+x, w) \wedge w<y \wedge \forall w^{\prime}\left(\lambda\left(x, z+x, w^{\prime}\right) \wedge w^{\prime}<y \rightarrow w^{\prime} \leq w\right)\right. \\
& \wedge(z=0 \rightarrow 10 w=2 x) \\
& \wedge\left(z \neq 0 \rightarrow \exists v, w^{\prime \prime}\left(H(x, z+x, v) \wedge \lambda\left(x, z, w^{\prime \prime}\right) \wedge w^{\prime \prime}<y\right.\right. \\
& \wedge \forall w^{\prime \prime \prime}\left(\lambda\left(x, z, w^{\prime \prime \prime}\right) \wedge w^{\prime \prime \prime}<y \rightarrow w^{\prime \prime \prime} \leq w^{\prime \prime}\right) \\
& \left.\left.\left.\wedge \pi\left(x, w-w^{\prime \prime}, v, 2 x\right)\right)\right)\right) .
\end{aligned}
$$

Though these formulas have the same interpretation in any e.c. dimension group, they yield an interpretation of second-order arithmetic only in certain special groups. If $\mathcal{G}$ is an $\omega$-e.s. dimension group and $0<b$ in $\mathcal{E}$, then

$$
\mathcal{E} \models \exists y \mathcal{g}(b, y),
$$


and in fact for every $a \in S$ there is $c \in G$ with

$$
P(b, c)=(\mathbb{Q} \cap(-\infty, N(a)), \mathbb{Q} \cap(N(a), \infty)) .
$$

These properties permit a translation into $\mathcal{Q}$ of second-order arithmetic as follows. As in [6, pp. 117-118], the language of second-order arithmetic ${ }^{8}$ features number variables $x, y, z, \ldots$ and set variables $X, Y, Z, \ldots$; predicate symbols for numerical identity, strict numerical inequality, and set membership; function symbols for addition and multiplication; and constant symbols $\dot{n}$ for natural numbers $n$. The atomic formulas are identities between numerical terms, inequalities between numerical terms, and formulas $t \in X$, where $t$ is a numerical term and $X$ is a set variable; and arbitrary formulas are built from atomic ones with the help of connectives and quantifiers. If $\psi\left(y_{1}, \ldots, y_{r}, V_{1}, \ldots, V_{s}\right)$ is an unnested formula, in the language of second-order arithmetic, that does not contain the variable $x$, let the Q-formula $\psi^{(x)}(\bar{y}, \bar{V})$ result from $\psi(\bar{y}, \bar{V})$ as follows. First, replace all occurrences of the constant symbol $\dot{n}$ by $n x$. Next, relativize all number quantifiers to $\mathbb{N} \cdot x$. Then, relativize all set quantifiers to $\mathcal{f}(x, \cdot)$; for example, if . . contains no set quantifiers, replace

$$
\forall V \ldots
$$

by

$$
\forall V(\mathcal{g}(x, V) \rightarrow \ldots)
$$

where $V$ is now viewed as a variable of $\mathcal{Q}$. Finally, replace all subformulas $r \cdot s=t$ by $\pi(x, r, s, t)$ and all subformulas $t \in Y$ by $\mu(x, Y, t)$. Arguing by induction on the complexity of formulas, one may show the following.

Lemma 6.3 Suppose that $b>0$ in the e.c. dimension group $\mathcal{E}$, and let $\psi\left(y_{1}, \ldots, y_{r}, V_{1}, \ldots, V_{s}\right)$ be an unnested formula, of second-order arithmetic, that does not contain the variable $x$. Suppose that $k_{1}, \ldots, k_{r} \in \mathbb{N}, A_{1}, \ldots, A_{s} \subseteq \mathbb{N}$, and for $j=1, \ldots, s, c_{j}$ makes in $\mathbb{Q} \cdot b$ the cut made by

$$
\sum_{i=0}^{\infty} \chi_{A_{j}}(i) 10^{-(i+1)^{2}}
$$

in $\mathbb{Q}$. If $\psi(\bar{y}, \bar{V})$ contains no set quantifiers, or if $\mathcal{E}$ is $\omega$-e.s., then

$$
\mathbb{N} \models \psi(\bar{k}, \bar{A}) \quad \text { iff } \quad g \models \psi^{(b)}\left(k_{1} b, \ldots, k_{r} b, \bar{c}\right) .
$$

This result has many corollaries.

Corollary 6.4 Second-order arithmetic is recursively isomorphic to $T^{\mathrm{F}}$.

Proof Given Lemma 6.1, one need show only that second-order arithmetic is oneone reducible to $T^{\mathrm{F}}$. Let $\mathcal{E}$ be an $\omega$-e.s. dimension group. Lemma 6.3 implies that for all unnested sentences $\psi$ of second-order arithmetic that do not contain $x$,

$$
\mathbb{N} \models \psi \quad \text { iff } \quad \mathcal{E} \models \forall x>0\left(\psi^{(x)}\right) .
$$

Since $T^{\mathrm{F}}=\operatorname{Th}(\mathscr{E})$, the desired conclusion follows.

Note that the infinite-forcing companion of the $\{+,-, \wedge, \vee, 0\}$-theory of $l$-groups has the same complexity (by [5, Proof of Theorem 21]).

Because $T^{\mathcal{Q}}$ has the joint embedding property, Corollary 6.4 combines with [6, Proposition 7.13] to imply the following. 
Corollary 6.5 No i.g. dimension group is analytical (i.e., has diagram definable in second-order arithmetic).

By contrast, a diagram for the $\omega_{1}$-e.s. dimension group $\left(\mathbb{Z}_{\omega_{1}}\right)_{\mathcal{F}}$ (see [14, Theorem 7.7]) may be defined in ZFC.

Theorem 7.17 of [6] immediately implies the next result.

Corollary 6.6 There are $2^{\aleph_{0}}$ e.c. dimension groups that are pairwise elementarily inequivalent.

Again, the e.c. $l$-groups enjoy the same property (see [5, Corollary 22]).

By following [6, proof of Theorem 16.17], one may determine the recursiontheoretic complexity of the theory of e.c. dimension groups.

Theorem 6.7 The theory $T^{\mathrm{e}}$ of e.c. dimension groups is a complete $\Pi_{1}^{1}$-set.

Proof $\quad T^{\mathrm{e}}$ is $\Pi_{1}^{1}$ by [6, Lemma 7.4(i)] and will be $\Pi_{1}^{1}$-complete if every $\Pi_{1}^{1}$-set $A$ is one-one reducible to $T^{\mathrm{e}}$. Because $A$ is $\Pi_{1}^{1}$, it may be defined in $\mathbb{N}$ by a second-order formula $\psi(y)=$

$$
\forall V \theta(y, V)
$$

in which $\theta(y, V)$ contains no set quantifiers (see Rogers [11, Exercise 16-10]). Without loss of generality $\psi(y)$ and every $\psi(\dot{n})$ are unnested and $\psi(y)$ does not contain the variable $x$. So one obtains an injective recursive map $n \in \mathbb{N} \mapsto \psi(\dot{n})^{(x)}$. The desired conclusion holds if

$$
n \in A \quad \text { iff } \quad \forall x>0\left(\psi(\dot{n})^{(x)}\right) \in T^{\mathrm{e}}
$$

for all $n \in \mathbb{N}$.

Suppose that $n \in A$. If $\mathscr{G}$ is an e.c. dimension group, one must show that $\mathcal{E} \models \forall x>0\left(\psi(\dot{n})^{(x)}\right)$. Since $\psi(\dot{n})$ is $\forall V \theta(\dot{n}, V), \psi(\dot{n})^{(x)}$ is

$$
\forall V\left(\mathscr{g}(x, V) \rightarrow \theta(\dot{n}, V)^{(x)}\right) .
$$

So to confirm that $\mathcal{E} \models \forall x>0\left(\psi(\dot{n})^{(x)}\right)$, suppose that $0<b$ in $\mathcal{E}, c \in G$, and

$$
\mathcal{E} \models \mathcal{g}(b, c) ;
$$

one must show that $\mathscr{E} \models \theta(\dot{n}, c)^{(b)}$. Because $\mathcal{E}$ is e.c., $c$ makes in $\mathbb{Q} \cdot b$ the cut made in $\mathbb{Q}$ by $N(d)$ for some $d \in S$. If

$$
D=\{k \in \mathbb{N}: d(k)=2\},
$$

then

$$
N(d)=\sum_{i=0}^{\infty} \chi_{D}(i) 10^{-(i+1)^{2}}
$$

and so by Lemma 6.3

$$
\mathbb{N} \models \theta(\dot{n}, D) \quad \text { iff } \quad \mathcal{E} \models \theta(\dot{n}, c)^{(b)} .
$$

Since $n \in A$, which is defined in $\mathbb{N}$ by $\forall V \theta(y, V), \mathbb{N} \models \theta(\dot{n}, D)$, and so $\mathcal{E} \models \theta(\dot{n}, c)^{(b)}$, as desired.

Assume now that $n \notin A$, and let $\mathscr{H}$ be any $\omega$-e.s. dimension group. Because $n \notin A$, which is defined in $\mathbb{N}$ by $\psi(y)$, Lemma 6.3 implies that

$$
\mathscr{H} \models \forall x>0\left(\neg \psi(\dot{n})^{(x)}\right)
$$


and so

$$
\mathscr{H} \not \forall \forall x>0\left(\psi(\dot{n})^{(x)}\right) .
$$

Thus $\forall x>0\left(\psi(\dot{n})^{(x)}\right)$ fails in at least one e.c. dimension group and so does not belong to $T^{\mathrm{e}}$.

\section{Recursive Approximation to f.g. Dimension Groups}

Though no f.g. dimension group is arithmetic (see Corollary 5.6), this section will answer a question of Macintyre by showing that most of the previously described special properties of f.g. dimension groups occur already in recursive e.c. dimension groups. Such groups will be manufactured with the help of the machinery behind finite forcing, and the first few results of this section show that certain forcing-theoretic notions are recursive.

As in Section 4, let $\mathcal{A}$ be the set of closed literals of $\mathcal{Q}_{W}$.

Lemma 7.1 Let $S \subseteq \mathcal{A}$ be finite, and let $S_{+} \subseteq S$ consist of the atomic sentences in $S$. For each $\varphi \in S \backslash S_{+}$let $S_{\varphi}=S_{+} \cup\{\varphi\}$.

(i) If $S=S_{+}$, then $S$ is a condition just in case $S$ may be satisfied in the ordered abelian group $\mathbb{Q}$.

(ii) If $S \neq S_{+}$, then $S$ is a condition just in case every $S_{\varphi}$ may be satisfied in the ordered abelian group $\mathbb{Q}$; in this case $S$ may be satisfied in $\mathbb{Q}^{\left|S \backslash S_{+}\right|}$.

Proof The argument follows that for Lemma 4.6, in which $\mathscr{D}$ may be replaced by $\mathbb{Q}$ because $S$ is finite.

One may therefore reach the following conclusion.

Corollary 7.2 The set of conditions for finite forcing is recursive. ${ }^{9}$

Proof Given a finite nonempty $S \subseteq \mathcal{A}$, one wants to decide whether or not $S$ is a condition. For nonempty $T \subseteq S$, let $\widehat{T}$ be the existential closure of the sentence obtained from $\bigwedge T$ by replacing each $c_{i} \in W$ by the corresponding variable $y_{i}$. If $S=S_{+}$, then $S$ is a condition just in case $\mathbb{Q} \models \widehat{S}$; if $S \neq S_{+}$, then $S$ is a condition just in case $\mathbb{Q} \models \widehat{S_{\varphi}}$ for every $\varphi \in S \backslash S_{+}$. Because the $\mathcal{Q}$-theory of $\mathbb{Q}$ is recursive, the desired conclusion follows.

The construction given below will show that certain infinitary sentences forced by the empty condition may be satisfied in recursive dimension groups. An important ingredient in the construction is the next lemma.

Lemma 7.3 Suppose that $\emptyset$ forces

$$
\bigwedge_{i \in \mathbb{N}} \forall \bar{x}_{i} \bigvee_{j \in \mathbb{N}} \exists \bar{y}_{i j} \varphi_{i j}\left(\bar{x}_{i}, \bar{y}_{i j}\right),
$$

where each $\varphi_{i j}\left(\bar{x}_{i}, \bar{y}_{i j}\right)$ is a quantifier-free first-order formula in the $m_{i}$ variables $\bar{x}_{i}$ and the $n_{i j}$ variables $\bar{y}_{i j}$. Let $s: \mathbb{N}^{2} \rightarrow \mathbb{N}$ send pairs $(i, j) \in \mathbb{N}^{2}$ to formulas $\forall \bar{x}_{i} \exists \bar{y}_{i j} \varphi_{i j}\left(\bar{x}_{i}, \bar{y}_{i j}\right)$. There are functions $f, g$, and $h$, recursive in $s$, that send any condition $p$, natural number $i$, and $m_{i}$-tuple $\bar{d}$ of closed $\mathcal{Q}_{W}$-terms to a condition

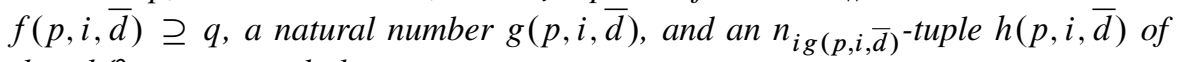
closed $\mathcal{Q}_{W}$-terms such that

$$
T^{\mathcal{Q}} \cup f(p, i, \bar{d}) \models \varphi_{i g(p, i, \bar{d})}(\bar{d}, h(p, i, \bar{d})) .
$$


Proof Theorems 3.4.4 and 2.3.4(f) of [7] imply that some $q \supseteq p$ forces some $\exists \bar{y}_{i j} \varphi_{i j}\left(\bar{d}, \bar{y}_{i j}\right)$. Thus $T^{\mathcal{Q}} \cup q \cup\left\{\exists \bar{y}_{i j} \varphi_{i j}\left(\bar{d}, \bar{y}_{i j}\right)\right\}$ has a model (see [7, Theorem 3.4.2]), $T^{\mathcal{Q}} \cup p \cup\left\{\exists \bar{y}_{i j} \varphi_{i j}\left(\bar{d}, \bar{y}_{i j}\right)\right\}$ has a model, and if $\bar{e}_{j}$ consists of the first $n_{i j}$ witnesses not in $p$ or $\bar{d}, T^{\mathcal{Q}} \cup p \cup\left\{\varphi_{i j}\left(\bar{d}, \bar{e}_{j}\right)\right\}$ has a model. One may effectively convert each $\varphi_{i j}$ to a formula $\bigvee_{k=1}^{r_{i j}} \varphi_{i j k}$ in disjunctive normal form, and $T^{\mathcal{Q}} \cup p \cup\left\{\varphi_{i j}\left(\bar{d}, \bar{e}_{j}\right)\right\}$ has a model just in case some $T^{\mathcal{Q}} \cup p \cup\left\{\varphi_{i j k}\left(\bar{d}, \bar{e}_{j}\right)\right\}$ has a model; that is, just in case the extension $q_{j k}$ of $p$ by the conjuncts of $\varphi_{i j k}\left(\bar{d}, \bar{e}_{j}\right)$ is a condition. One may enumerate all the $q_{j k}$ recursively in $s$, and Corollary 7.2 allows one effectively to check which of these are conditions. By hypothesis one of them is a condition, and if $q_{j_{0} k_{0}}$ is the first of these to be encountered, one may let $f(p, i, \bar{d})=q_{j_{0} k_{0}}, g(p, i, \bar{d})=j_{0}$, and $h(p, i, \bar{d})=\bar{e}_{j_{0}}$.

Now one may build models of infinitary sentences.

Theorem 7.4 If $\emptyset$ forces a sentence $(\star)=$

$$
\bigwedge_{i \in \mathbb{N}} \forall \bar{x}_{i} \bigvee_{j \in \mathbb{N}} \exists \bar{y}_{i j} \varphi_{i j}\left(\bar{x}_{i}, \bar{y}_{i j}\right)
$$

as in Lemma 7.3, then this sentence is true in some e.c. dimension group recursive in the function $s:(i, j) \in \mathbb{N}^{2} \mapsto \forall \bar{x}_{i} \exists \bar{y}_{i j} \varphi_{i j}\left(\bar{x}_{i}, \bar{y}_{i j}\right)$.

Proof There is an axiom $(\star)^{\prime}$, of the same shape as $(\star)$, whose associated function is recursive and which axiomatizes the class of e.c. dimension groups (see [14, Corollary 3.6]). Because $T^{\mathcal{Q}}$ is $\forall \exists, \emptyset$ forces $(\star)^{\prime}$ (see [7, Corollary 3.4.3]). So by conjoining $(\star)$ with $(\star)^{\prime}$ one reduces the present problem to the following: show that $(\star)$ has a model recursive in $s$.

Let $a$ be a recursive enumeration of $\mathcal{A}$, and let $(k, l) \mapsto \bar{d}_{k l}$ be a recursive enumeration of all finite sequences of closed $\mathcal{Q}_{W}$-terms, where $l \mapsto \bar{d}_{k l}$ enumerates the $k$-tuples of such terms. When $\varphi \in \mathcal{A}$, let $-\varphi$ be $\neg \varphi$, if $\varphi$ is atomic, and $\psi$ if $\varphi$ is $\neg \psi$. The following discussion will describe a function $i \in \mathbb{N} \mapsto S^{(i)}$, recursive in $s$, such that each $S^{(i)}$ is a condition and each $S^{(i)} \subseteq S^{(i+1)} ; \bigcup_{i \in \mathbb{N}} S^{(i)}$ will be the diagram of the model under construction.

Let $S^{(0)}=\varnothing$.

Given the condition $S^{(i)}$, let

$$
S^{(i) \prime}= \begin{cases}S^{(i)} \cup\{a(i)\} & \text { if this set is a condition, } \\ S^{(i)} \cup\{-a(i)\} & \text { otherwise. }\end{cases}
$$

Applying Lemma 7.3 to the tuples $\bar{d}_{m_{k}} l$ with $k, l \leq i$, one obtains a condition $S^{(i+1)} \supseteq S^{(i) \prime}$, integers $j_{k l}$ with $k, l \leq i$, and tuples $\bar{e}_{k l}$ of closed $\mathcal{Q}_{W}$-terms such that

$$
T^{\mathcal{Q}} \cup S^{(i+1)} \models \bigwedge_{k, l \leq i} \varphi_{k j_{k l}}\left(\bar{d}_{m_{k} l}, \bar{e}_{k l}\right) .
$$

Letting $S^{(\omega)}=\bigcup_{i \in \mathbb{N}} S^{(i)}$, one sees that $S^{(\omega)}$ is recursively enumerable in $s$. Since $i \in \mathbb{N} \mapsto S^{(i)}$ is a nondecreasing sequence of conditions, $T^{\mathcal{Q}} \cup S^{(\omega)}$ is consistent; since $a(i)$ or $-a(i)$ belongs to $S^{(i+1)}$, every atomic $\mathcal{Q}_{W}$-sentence or its negation belongs to $S^{(\omega)}$. Thus $S^{(\omega)}$, the diagram of a $\mathcal{Q}$-substructure $\mathcal{M}$ of a model $\mathcal{N}$ of $T^{\mathcal{Q}}$, is recursive in $s$. The structure $\mathcal{M}$ also satisfies $(\star)$ : for if $k \in \mathbb{N}$ and $\bar{r}$ is 
an $m_{k}$-tuple of closed $\mathcal{Q}_{W}$-terms, then $\bar{r}=\bar{d}_{m_{k} l}$ for some $l$, and if $i=\max \{k, l\}$, then there are an integer $j_{k l}$ and a tuple $\bar{e}_{k l}$ of closed $\mathcal{Q}_{W}$-terms with

$$
T^{\mathcal{Q}} \cup S^{(i+1)} \models \varphi_{k j_{k l}}\left(\bar{d}_{m_{k} l}, \bar{e}_{k l}\right) .
$$

Since $\mathcal{N} \models T^{\mathcal{Q}} \cup S^{(i+1)}, \mathcal{N} \models \varphi_{k j_{k l}}\left(\bar{r}, \bar{e}_{k l}\right)$, and so $\mathcal{M} \models \varphi_{k j_{k l}}\left(\bar{r}, \bar{e}_{k l}\right)$ because the sentence is quantifier-free. The desired conclusion follows because every element of $M$ is named by a closed $\mathcal{Q}_{W}$-term.

The results of $[14$, Section 6] and of Sections 2 and 3 above show that there is a sentence $(\star)$, forced by $\emptyset$, whose function $s$ is recursive and which is true just in e.c. dimension groups that are simple, that obey

$$
\forall z>0 \forall x(\forall w \in \mathbb{Q} \cdot z(x \nmid w) \rightarrow x \in \mathbb{Q} \cdot z)
$$

and

$$
\begin{aligned}
\forall x & >0 \forall y \forall z \in \mathbb{Q} \cdot x[(y<z \rightarrow \exists w \in \mathbb{Q} \cdot x(y<w<z)) \\
& \wedge(z<y \rightarrow \exists w \in \mathbb{Q} \cdot x(z<w<y)) \\
& \wedge(y \mid z \rightarrow \exists v, w \in \mathbb{Q} \cdot x(v<z<w \wedge y \mid v, w))],
\end{aligned}
$$

that admit no proper self-embeddings, and whose automorphism groups consist of multiplications by positive rationals. Thus some recursive e.c. dimension group enjoys all these properties. Though they occur in all f.g. dimension groups, no such group is arithmetic; so these properties fail to distinguish between f.g. and suitable recursive e.c. dimension groups. By contrast, [12, Section 2] and [4, Theorem 1.8] show that the f.g. abelian $l$-groups are the e.c. abelian $l$-groups that satisfy a particular first-order sentence: a sentence equivalent, in e.c. abelian $l$-groups, to a $\{+,-, \wedge, \vee, 0\}$-sentence of the form $(\star)$. Only the results of Section 5 begin to distinguish f.g. from recursive e.c. dimension groups by revealing features of f.g. dimension groups incompatible with an arithmetic diagram.

Note finally that the class of f.g. dimension groups is the class of models of a sentence

$$
\bigwedge_{i \in \mathbb{N}} \forall \bar{x}_{i} \bigvee_{j \in \mathbb{N}} \exists \bar{y}_{i j} \varphi_{i j}\left(\bar{x}_{i}, \bar{y}_{i j}\right)
$$

as in Lemma 7.3 (see [7, Exercises 4.3.3-4.3.4]); so Theorem 7.4 and Corollary 5.6 imply that every such sentence produces a nonarithmetic $(i, j) \mapsto \forall \bar{x}_{i} \exists \bar{y}_{i j} \varphi_{i j}\left(\bar{x}_{i}\right.$, $\left.\bar{y}_{i j}\right)$. By following the hints in [7, Exercises 4.3.3-4.3.4], one may obtain a sentence whose function $(i, j) \mapsto \forall \bar{x}_{i} \exists \bar{y}_{i j} \varphi_{i j}\left(\bar{x}_{i}, \bar{y}_{i j}\right)$ is recursive in the relation " $p$ forces $\psi$," which one may show is recursive in first-order arithmetic. So one may establish the next result.

Corollary 7.5 There is an f.g. dimension group recursive in first-order arithmetic.

Proof Let $\Vdash_{\text {HW }}$ be the finite-forcing relation used in [6]. Lemma A.1 of the Appendix says that for all conditions $p$ and first-order $\mathcal{Q}_{W}$-sentences $\psi$ with connectives and quantifiers among $\wedge, \vee, \neg$, and $\exists$,

$$
p \text { forces } \psi \quad \text { iff } \quad p \Vdash_{\mathrm{HW}} \neg \neg \psi \text {. }
$$

So if $\Vdash_{\text {HW }}$ is recursive in first-order arithmetic, the desired conclusion follows.

Relying on the recursive encoding of the syntax of $\mathcal{Q}_{W}$, one may define a recursive sequence $\left\{\varphi_{n}^{1}(x, y)\right\}_{n \geq 0}$, of formulas of first-order arithmetic, such that $\varphi_{n}^{1}(x, y)$ defines the set of pairs $(\ulcorner p\urcorner, \theta)$, where $\ulcorner p\urcorner$ is the Gödel number of a condition $p$, 
$\theta$ is (the Gödel number of) a $\mathcal{Q}_{W}$-sentence of complexity at most $n$, and $p \Vdash_{\text {HW }} \theta$. If such a sequence exists, $\vdash_{\mathrm{HW}}$ will be recursive in first-order arithmetic. Because $\left\{(\ulcorner p\urcorner, \theta): p\right.$ is a condition and $\theta$ is an atomic $\mathcal{Q}_{W}$-sentence and $\left.\theta \in p\right\}$ is recursive, a suitable $\varphi_{1}^{1}(x, y)$ exists; and if $\varphi_{n}^{1}(x, y)$ is available, one may let $\varphi_{n+1}^{1}(x, y)$ be a rendition of

$$
\begin{aligned}
& \text { (" } \left.y \text { is a } \mathcal{Q}_{W} \text {-sentence of complexity } \leq n " \wedge \varphi_{n}^{1}(x, y)\right) \\
& \vee\left[" y \text { is a } \mathcal{Q}_{W} \text {-sentence of complexity } n+1 "\right. \\
& \wedge\left\{\left(" y \text { is a disjunction } \theta \vee \gamma " \wedge\left[\varphi_{n}^{1}(x, \theta) \vee \varphi_{n}^{1}(x, \gamma)\right]\right)\right. \\
& \vee\left(" y \text { is a conjunction } \theta \wedge \gamma " \wedge\left[\varphi_{n}^{1}(x, \theta) \wedge \varphi_{n}^{1}(x, \gamma)\right]\right) \\
& \vee(" y \text { is an existential quantification } \exists x \psi(x) " \\
& \left.\wedge \exists t\left(t \text { is a closed instance of } y \wedge \varphi_{n}^{1}(x, t)\right)\right) \\
& \vee(" y \text { is a negation } \neg \theta " \\
& \left.\left.\left.\wedge \forall z\left[z \text { is a code of a condition extending } x \rightarrow \neg \varphi_{n}^{1}(z, \theta)\right]\right)\right\}\right] .
\end{aligned}
$$

The proof of Corollary 7.5 suggests a more straightforward method of producing an f.g. dimension group recursive in first-order arithmetic. Namely, if $\left\{\theta_{n}\right\}_{n \in \mathbb{N}}$ is a recursive enumeration of all $\mathcal{Q}_{W}$-sentences, define the sequence $\left\{p_{n}\right\}_{n \in \mathbb{N}}$ of conditions as follows: $p_{0}=\emptyset$; given $p_{n}$, let $p_{n+1}$ be the least condition-in a fixed recursive enumeration of the conditions - that extends $p_{n}$ and bears $\Vdash_{H W}$ to $\theta_{n}$ or to $\neg \theta_{n}$. Since $\Vdash_{H W}$ is recursive in first-order arithmetic, so is the sequence $\left\{p_{n}\right\}_{n \in \mathbb{N}}$, whose union will be the diagram of an f.g. dimension group. The next section will show that one may emulate this procedure to produce an i.g. dimension group recursive in second-order arithmetic if one treats i.g. dimension groups in terms of a notion of forcing with countable conditions. Of course, one will need a method of extending a given condition to a new one that forces $\theta_{n}$ or $\neg \theta_{n}$, and the most easily described guarantee of such a method would assume that the power set of the natural numbers has an analytical well-ordering.

\section{Hyperarithmetic Approximation to i.g. Dimension Groups}

By establishing an analogue of Theorem 7.4 for i.g. dimension groups, this section will show that they are not characterized by the previously isolated properties of such groups: properties that may be realized in a single e.c. dimension group whose recursion-theoretic complexity is too low. The form of Theorem 7.4 and the last argument of Section 7 suggest that one develop i.g. structures in terms of a notion of forcing recursive in second-order arithmetic. I.g. structures were originally defined in terms of a notion of forcing with structures as conditions (see, e.g., [6, Chapter 3]). Because these structures may have arbitrary cardinality, this notion of forcing does not directly meet present requirements; but by restricting attention to conditions that are diagrams of countable structures one obtains a notion of forcing, instances of which may be defined in second-order arithmetic. In terms of this notion one can prove versions of Lemma 7.3 and Theorem 7.4 and show also that if the power set of the natural numbers has an analytical well-ordering, then there is an i.g. dimension group recursive in second-order arithmetic.

To define the forcing, with countable conditions, exploited below, let $(r, s) \in$ $\mathbb{N}^{2} \mapsto c_{r, s}$ be a bijection of $\mathbb{N}^{2}$ onto the set $W$ of witnesses, and call $c_{r, s}$ a witness 
of level $r$. If $F \subseteq \mathbb{N}$ is finite, let

$$
\mathcal{Q}_{F}=\mathcal{Q} \cup\left\{c_{r, s}: r \in F \text { and } s \in \mathbb{N}\right\} .
$$

An $F$-condition is the $\mathcal{Q}_{F}$-diagram of a model of $T_{\forall}^{\mathcal{Q}}$; so $S$ is an $F$-condition just in case $S$ consists of closed literals of $\mathcal{Q}_{F}, \varphi \in S$ or $(\neg \varphi) \in S$ for every atomic sentence $\varphi$ of $\mathcal{Q}_{F}$, and every finite subset of $S$ is a condition in the sense of finite forcing. A condition is a set that is an $F$-condition for some finite $F \subseteq \mathbb{N}$, and the set of conditions is partially ordered by set-theoretic inclusion. So $\mathbb{O}$, the unique $\emptyset$-condition, is the least condition in this partial ordering. When $S$ is a condition, let $L(S)$ be the unique finite $F \subseteq \mathbb{N}$ for which $S$ is an $F$-condition.

Much as in [7, Section 2.3], a sequence $\bar{S}=\left\{S_{i}\right\}_{i \in \mathbb{N}}$ of conditions with each $S_{i} \subseteq S_{i+1}$ is called a construction sequence. $\bigcup \bar{S}=\bigcup_{i \in \mathbb{N}} S_{i}$ will be the diagram of a $\mathcal{Q}$-structure $A^{+}(\bar{S})$, called the compiled structure. If $X$ is an infinite and coinfinite subset of $\mathbb{N} \backslash\{0\}$ and $P$ is a property of sets $\bigcup \bar{S}$, one may define a two-person game $G(P, X)$ with players $\forall$ and $\exists$. They take turns building a construction sequence $\bar{S}=\left\{S_{i}\right\}_{i \in \mathbb{N}}-\exists(\forall)$ picking $S_{i}$ when $i \in X(i \in \mathbb{N} \backslash X)$-and $\exists(\forall)$ wins the play $\bar{S}$ of the game just in case $\bigcup \bar{S}$ has property $P$. If $R$ is a condition, $R$ is said to force $P$ just in case $\exists$ has a strategy that will win $G(P, X)$ from any position $\left(S_{0}, \ldots, S_{k}\right)$ with $R \subseteq S_{k}$. When $\varphi$ is a sentence of $\left(\mathcal{Q}_{W}\right)_{\omega_{1} \omega}$ and $R$ is a condition, $R$ is said to force $\varphi$ just in case $R$ forces the property $A^{+}(\bar{S}) \models \varphi$ of $\bigcup \bar{S}$.

These definitions do not quite agree with the definitions of [7, Chapter 2]; for example, the adjunction to a condition of a single closed literal, even if consistent with $T_{\forall}^{\mathcal{Q}}$, need not be a condition. But as the Appendix shows, many results analogous to those of finite forcing continue to hold, including the next result.

Theorem 8.1 Let $P$ be an $F$-condition, and let $\psi$ be the $\left(\mathcal{Q}_{F}\right)_{\omega_{1} \omega^{- \text {sentence }}}$

$$
\forall \bar{x} \bigvee_{i \in \mathbb{N}} \exists \bar{y}_{i} \bigwedge_{j \in \mathbb{N}} \varphi_{i j}\left(\bar{x}, \bar{y}_{i}\right)
$$

where each $\varphi_{i j}\left(\bar{x}, \bar{y}_{i}\right)$ is a quantifier-free first-order formula; then $P$ forces $\psi$ just in case for all closed terms $\bar{c}$ and all conditions $Q \supseteq P$ there are $i \in \mathbb{N}$ and witnesses $\bar{w}_{i}$ for which $T_{\forall}^{\mathcal{Q}} \cup Q \cup\left\{\varphi_{i j}\left(\bar{c}, \bar{w}_{i}\right): j \in \mathbb{N}\right\}$ is satisfiable.

In familiar fashion one may give a uniformly primitive-recursive treatment of the syntax of the languages $\mathcal{Q}_{W}$ and $\mathcal{Q}_{F}$. In what follows, first-order formulas will be viewed as their Gödel numbers. When $F \subseteq \mathbb{N}$ is finite, $\ulcorner F\urcorner$ is the Gödel number of $F$ : a number from which membership in $\bar{F}$, and the number of elements of $F$, may be recursively determined. When $\bar{d}$ is a finite tuple of closed $\mathcal{Q}_{W}$-terms, $\ulcorner\bar{d}\urcorner$ will be its Gödel number.

Ordering the closed literals of $\mathcal{Q}_{W}$ by increasing Gödel number, one obtains an induced ordering of the set of all closed literals from $\mathcal{Q}_{F}$. One may use this ordering to define, recursively in $\ulcorner F\urcorner$, a binary branching tree of finite sequences of closed literals of $\mathcal{Q}_{F}$. If the closed literals of $\mathcal{Q}_{F}$ are ordered as $\varphi_{0}<\varphi_{1}<\varphi_{2}<\cdots$, nodes at the $n$th level of the tree are finite sequences $\left\langle\psi_{0}, \ldots, \psi_{n}\right\rangle$, where each $\psi_{i}= \pm \varphi_{i}$ (notation as in Section 7); and the immediate descendants of this node in the tree are the sequences $\left\langle\psi_{0}, \ldots, \psi_{n}, \varphi_{n+1}\right\rangle$ and $\left\langle\psi_{0}, \ldots, \psi_{n},-\varphi_{n+1}\right\rangle$, where the first is considered to be to the left of the second and to the left of any node $\left\langle\theta_{0}, \ldots, \theta_{n}, \theta_{n+1}\right\rangle$ with $\left\langle\psi_{0}, \ldots, \psi_{n}\right\rangle$ to the left of $\left\langle\theta_{0}, \ldots, \theta_{n}\right\rangle$. These trees, and the associated orderings of levels, may all be defined recursively in $\ulcorner F\urcorner$. 
A set $A$ of closed literals of $Q_{F}$ is an $F$-condition just in case every finite subset of $A$ is consistent with $T_{\forall}^{\mathcal{Q}}$ and for every closed literal $\varphi$ of $\mathcal{Q}_{F}, \varphi \in A$ or $-\varphi \in A$. Since consistency with $T_{\forall}^{\mathcal{Q}}$ of a given finite set of quantifier-free sentences of $\mathcal{Q}_{W}$ may be checked recursively (see Corollary 7.2), there is a $\Pi_{1}^{0}$-formula $C(X, y)$ that defines the set of pairs $(A,\ulcorner F\urcorner)$, where $A$ is an $F$-condition. ${ }^{10}$ Every $F$-condition $A$ determines a unique path in the tree of $Q_{F}$-literals: the path $\emptyset,\left\langle\psi_{0}\right\rangle,\left\langle\psi_{0}, \psi_{1}\right\rangle, \ldots$, where $\psi_{i}$ is $\varphi_{i}\left(-\varphi_{i}\right)$ just in case $\varphi_{i}\left(-\varphi_{i}\right)$ belongs to $A$. If $B$ is a set of quantifier-free sentences of $\mathcal{Q}_{F}$ that is consistent with $T_{\forall}^{\mathcal{Q}}, B$ will be implied by at least one $F$-condition. The $F$-condition $A$, implying $B$, whose path is leftmost in the tree of closed $Q_{F}$-literals may be defined in terms of the nodes on its path: $\left\langle\psi_{0}, \ldots, \psi_{n}\right\rangle$ is such a node just in case $T_{\forall}^{\mathcal{Q}} \cup B \cup\left\{\psi_{0}, \ldots, \psi_{n}\right\}$ is consistent but $T_{\forall}^{\mathcal{Q}} \cup B \cup\left\{\theta_{0}, \ldots, \theta_{n}\right\}$ is inconsistent whenever $\left\langle\theta_{0}, \ldots, \theta_{n}\right\rangle$ is to the left of $\left\langle\psi_{0}, \ldots, \psi_{n}\right\rangle$. When $D$ is a set of quantifier-free sentences of $\mathcal{Q}_{W}$, consistency of $T_{\forall}^{\mathcal{Q}} \cup D$ is $\Pi_{1}^{0}$ in $D$. So there is a $\Sigma_{3}^{0}$-formula $E(X, y, z)$ with the following property for all $B \subseteq \mathbb{N}$ : if the set $B^{\prime}$ of quantifier-free sentences of $\mathcal{Q}_{F}$ in $B$ is consistent with $T_{\forall}^{\mathcal{Q}}, E(B,\ulcorner F\urcorner, z)$ defines the $F$-condition $A$, implying $B^{\prime}$, with leftmost path; otherwise $E(B,\ulcorner F\urcorner, z)$ defines $\emptyset$.

One may now state an analogue of Lemma 7.3.

Lemma 8.2 In the language of second-order arithmetic there are a $\Delta_{1}^{0}$-formula $F_{1}(z, w, v, x)$, a $\Sigma_{4}^{0}$-formula $F_{2}(X, z, w, Y, t, v, x), a \Delta_{2}^{0}$-formula $G(X, z, w, Y, t$, $v, x)$, and $a \Delta_{2}^{0}$-formula $H(X, z, w, Y, t, v, x)$ with the following property. Suppose that $S$ is the graph ${ }^{11}$ of a function

$$
(i, j, k) \in \mathbb{N}^{3} \mapsto \forall \bar{x}_{i} \exists \bar{y}_{i j} \varphi_{i j k}\left(\bar{x}_{i}, \bar{y}_{i j}\right),
$$

where each $\varphi_{i j k}\left(\bar{x}_{i}, \bar{y}_{i j}\right)$ is a quantifier-free first-order $\mathcal{Q}$-formula in the $m_{i}$ variables $\bar{x}_{i}$ and the $n_{i j}$ variables $\bar{y}_{i j}$; $\mathbb{O}$ forces

$$
\bigwedge_{i \in \mathbb{N}} \forall \bar{x}_{i} \bigvee_{j \in \mathbb{N}} \exists \bar{y}_{i j} \bigwedge_{k \in \mathbb{N}} \varphi_{i j k}\left(\bar{x}_{i}, \bar{y}_{i j}\right)
$$

$P$ is an $F$-condition; $i, b \in \mathbb{N}$; and $\bar{d}$ is an $m_{i}$-tuple of closed $\mathcal{Q}_{W}$-terms. Then there is a unique $c \in \mathbb{N}$ with

$$
F_{1}(\ulcorner F\urcorner, b,\ulcorner\bar{d}\urcorner, c) ;
$$

$c=\left\ulcorner F^{\prime}\right\urcorner$ for some finite $F^{\prime} \subseteq \mathbb{N}$ with $F \cup\{b\} \subseteq F^{\prime}$, and the $d s$ are closed $\mathcal{Q}_{F^{\prime}}$-terms; $F_{2}(S,\ulcorner F\urcorner, b, P, i,\ulcorner\bar{d}\urcorner, x)$ defines an $F^{\prime}$-condition $B \supseteq P$; there is a unique $j \in \mathbb{N}$ with

$$
G(S,\ulcorner F\urcorner, b, P, i,\ulcorner\bar{d}\urcorner, j) ;
$$

there is a unique $l \in \mathbb{N}$ with

$$
H(S,\ulcorner F\urcorner, b, P, i,\ulcorner\bar{d}\urcorner, l) ;
$$

and $l=\ulcorner\bar{e}\urcorner$ for some $n_{i j}$-tuple $\bar{e}$ of closed $\mathcal{Q}_{F^{\prime}}$-terms with

$$
B \models \bigwedge_{k \in \mathbb{N}} \varphi_{i j k}(\bar{d}, \bar{e}) .
$$

Proof Since $P$ forces

$$
\bigwedge_{i \in \mathbb{N}} \forall \bar{x}_{i} \bigvee_{j \in \mathbb{N}} \exists \bar{y}_{i j} \bigwedge_{k \in \mathbb{N}} \varphi_{i j k}\left(\bar{x}_{i}, \bar{y}_{i j}\right)
$$


there are $j \in \mathbb{N}$ and witnesses $\bar{w}_{i j}$ such that

$$
T_{\forall}^{\mathcal{Q}} \cup P \cup\left\{\varphi_{i j k}\left(\bar{d}, \bar{w}_{i j}\right): k \in \mathbb{N}\right\}
$$

is finitely satisfiable. If $r$ is the least natural number not in

$$
F \cup\{b\} \cup \text { the set of levels of witnesses in } \bar{d} \text {, }
$$

the next-to-last display is finitely satisfiable just in case

$$
T_{\forall}^{\mathcal{Q}} \cup P \cup\left\{\varphi_{i j k}\left(\bar{d}, c_{r, 0}, \ldots, c_{r, n_{i j}-1}\right): k \in \mathbb{N}\right\}
$$

is finitely satisfiable. There is a $\Delta_{1}^{0}$-formula $F_{0}(z, w, t, v, x)$ such that for $\ulcorner F\urcorner, b, i$, $\ulcorner\bar{d}\urcorner$ as above, $F_{0}(\ulcorner F\urcorner, b, i,\ulcorner\bar{d}\urcorner, x)$ defines $r$. So since the set of conditions for finite forcing is recursive (see Corollary 7.2), there is a $\Pi_{1}^{0}$-formula $K(X, z, w, Y, t, v, x)$ such that for all $S,\ulcorner F\urcorner, b, P, i,\ulcorner\bar{d}\urcorner$ as above, $K(S,\ulcorner F\urcorner, b, P, i,\ulcorner\bar{d}\urcorner, x)$ defines the set of all $j \in \mathbb{N}$ for which the last display is finitely satisfiable. Thus there is a $\Delta_{2}^{0}$-formula $G(X, z, w, Y, t, v, x)$ such that for all $S,\ulcorner F\urcorner, b, P, i,\ulcorner\bar{d}\urcorner$ as above, $G(S,\ulcorner F\urcorner, b, P, i,\ulcorner\bar{d}\urcorner, x)$ defines the least $j \in \mathbb{N}$ for which the last display is finitely satisfiable (and so satisfiable). Let $F_{1}(z, w, t, v, x)$ be a $\Delta_{1}^{0}$-formula such that for $\ulcorner F\urcorner, b, i,\ulcorner\bar{d}\urcorner$ as above, $F_{1}(\ulcorner F\urcorner, b, i,\ulcorner\bar{d}\urcorner, x)$ defines $\left\ulcorner F^{\prime}\right\urcorner$, where

$$
F^{\prime}=F \cup\{b\} \cup \text { the set of levels of witnesses in } \bar{d} \cup\{r\} .
$$

With the help of $G$ and $F_{1}$ one may write down a $\Delta_{2}^{0}$-formula $H(X, z, w, Y, t, v, x)$ such that for all $S,\ulcorner F\urcorner, b, P, i,\ulcorner\bar{d}\urcorner$ as above $H(S,\ulcorner F\urcorner, b, P, i,\ulcorner\bar{d}\urcorner, x)$ defines the unique $l$ of the form $\left\ulcorner\left(c_{r, 0}, \ldots, c_{r, n_{i j}-1}\right)\right\urcorner$, where $r$ is the unique element of

$$
F^{\prime} \backslash(F \cup\{b\} \cup \text { the set of levels of witnesses in } \bar{d})
$$

and $G(S,\ulcorner F\urcorner, b, P, i,\ulcorner\bar{d}\urcorner, j)$. There is a $\Sigma_{1}^{0}$-formula $U\left(X, Y, t_{1}, t_{2}, t_{3}, v, x\right)$ such that for all $S, P, i, j, r,\ulcorner\bar{d}\urcorner$ as above, $U(S, P, i, j, r,\ulcorner\bar{d}\urcorner, x)$ defines $P \cup\left\{\varphi_{i j k}(\bar{d}\right.$, $\left.\left.c_{r, 0}, \ldots, c_{r, n_{i j}-1}\right): k \in \mathbb{N}\right\}$. In the formula $E(X, y, x)$ described before the statement of Lemma 8.2, replace all subformulas $w \in X$ by $U\left(X, Y, t_{1}, t_{2}, t_{3}, v, w\right)$ to obtain the formula $E^{\prime}\left(X, Y, t_{1}, t_{2}, t_{3}, v, y, x\right)$. For $S, P, i, j, r,\ulcorner\bar{d}\urcorner, F^{\prime}$ as above, $E^{\prime}\left(S, P, i, j, r,\ulcorner\bar{d}\urcorner,\left\ulcorner F^{\prime}\right\urcorner, z\right)$ defines the $F^{\prime}$-condition, implying $P \cup\left\{\varphi_{i j k}\left(\bar{e}, c_{r, 0}\right.\right.$, $\left.\left.\ldots, c_{r, n_{i j}-1}\right): k \in \mathbb{N}\right\}$, with leftmost path (if $T_{\forall}^{\mathcal{Q}} \cup P \cup\left\{\varphi_{i j k}\left(\bar{d}, c_{r, 0}, \ldots, c_{r, n_{i j}-1}\right)\right.$ : $k \in \mathbb{N}$ \} is consistent; otherwise $\emptyset$ is defined). Because $E$ is $\Sigma_{3}^{0}$ and $U$ is $\Sigma_{1}^{0}, E^{\prime}$ will be $\Sigma_{4}^{0}$. Finally, let $F_{2}(X, z, w, Y, t, v, x)$ be

$$
\begin{aligned}
& \exists t_{2} \exists t_{3} \exists y\left[G\left(X, z, Y, t, v, t_{2}\right) \wedge F_{0}\left(X, z, w, Y, t, v, t_{3}\right)\right. \\
& \left.\wedge F_{1}(X, z, w, Y, t, v, y) \wedge E^{\prime}\left(X, Y, t, t_{2}, t_{3}, v, y, x\right)\right] .
\end{aligned}
$$

Since $G$ is $\Delta_{2}^{0}, F_{0}$ and $F_{1}$ are $\Delta_{1}^{0}$, and $E^{\prime}$ is $\Sigma_{4}^{0}, F_{2}$ is $\Sigma_{4}^{0}$.

A version of Theorem 7.4 goes as follows.

Theorem 8.3 If $\mathbb{O}$ forces a sentence

$$
\bigwedge_{i \in \mathbb{N}} \forall \bar{x}_{i} \bigvee_{j \in \mathbb{N}} \exists \bar{y}_{i j} \bigwedge_{k \in \mathbb{N}} \varphi_{i j k}\left(\bar{x}_{i}, \bar{y}_{i j}\right)
$$

as in Lemma 8.2, then this sentence is true in some model of $T_{\forall}^{\mathcal{Q}}$-hyperarithmetic in the function $S:(i, j, k) \in \mathbb{N}^{3} \mapsto \forall \bar{x}_{i} \exists \bar{y}_{i j} \varphi_{i j k}\left(\bar{x}_{i}, \bar{y}_{i j}\right)$. 
Proof Much as in the proof of Theorem 7.4, one defines an $S$-recursive sequence of arithmetical definitions of conditions $S^{0} \subseteq S^{1} \subseteq \ldots$ that imply larger and larger pieces of the infinitary sentence. Let $(k, l) \mapsto \bar{d}_{k l}$ be as in the proof of Theorem 7.4, let $a: \mathbb{N} \rightarrow \mathbb{N}$ be a recursive surjection such that $a^{-1}\{i\}$ is infinite for every $i \in \mathbb{N}$, and let $b: \mathbb{N} \rightarrow \mathbb{N}$ be the recursive function with

$$
b(i)=|\{k<i: a(k)=a(i)\}|
$$

for all $i \in \mathbb{N}$. Using the formula $F_{1}(z, w, v, x)$ from Lemma 8.2, one may define an $S$-recursive function $c: \mathbb{N} \rightarrow \mathbb{N}$ such that

$$
F_{1}\left(\ulcorner\emptyset\urcorner, 0,\left\ulcorner\bar{d}_{m_{a(0)} b(0)}\right\urcorner, c(0)\right)
$$

and

$$
F_{1}\left(c(i), i,\left\ulcorner\bar{d}_{m_{a(i+1)} b(i+1)}\right\urcorner, c(i+1)\right)
$$

for all $i \in \mathbb{N}$; each $c(i)$ will be $\left\ulcorner F_{i}\right\urcorner$, where $L\left(S^{i}\right)=F_{i}$. One may now define an $S$-recursive sequence $\left\{\varphi_{i}(X, Y, z)\right\}_{i \in \mathbb{N}}$, of formulas of second-order arithmetic, with the idea that $\varphi_{i}(S, \mathbb{O}, z)$ will define $S^{i}: \varphi_{0}(X, Y, z)$ is $F_{2}(X,\ulcorner\emptyset\urcorner, 0, Y, a(0)$, $\left.\left\ulcorner\bar{d}_{m_{a(0)} b(0)}\right\urcorner, z\right)$, and for every $i \in \mathbb{N}, \varphi_{i+1}(X, Y, z)$ is

$$
F_{2}\left(X, c(i), i, \varphi_{i}(X, Y, \ldots), a(i+1),\left\ulcorner\bar{d}_{m_{a(i+1)} b(i+1)}\right\urcorner, z\right),
$$

the result of replacing every occurrence of

$$
-\in Y
$$

in $F_{2}\left(X, c(i), i, Y, a(i+1),\left\ulcorner\bar{d}_{m_{a(i+1)} b(i+1)}\right\urcorner, z\right)$, by

$$
\varphi_{i}(X, Y, \ldots) \text {. }
$$

Since $F_{2}$ is $\Sigma_{4}^{0}$, one may show by induction that $\varphi_{i}(X, Y, z)$ is $\Delta_{5(i+1)}^{0}$. Because every condition forces the infinitary sentence displayed in the statement of the theorem, one may show by induction that for every $i, \varphi_{i}(S, \mathbb{O}, z)$ defines an $F_{i}$-condition $S^{i} \subseteq S^{i+1}$ with $\left\ulcorner F_{i}\right\urcorner=c(i)$, that $i \in F_{i} \subseteq F_{i+1}$ always, and that for each $i$ there are $j \in \mathbb{N}$ and an $n_{a(i) j}$-tuple $\bar{e}$ of witnesses from $\mathcal{Q}_{F_{i}}$ with

$$
S^{i} \models \bigwedge_{k \in \mathbb{N}} \varphi_{a(i) j k}\left(\bar{d}_{m_{a(i)} b(i)}, \bar{e}\right) .
$$

So $S^{\omega}=\bigcup_{i \in \mathbb{N}} S^{i}$ will be the $\mathcal{Q}_{W}$-diagram of a $\mathcal{Q}_{W}$-structure $\mathcal{M} \models T_{\forall}^{\mathcal{Q}}$. If $i^{\prime} \in \mathbb{N}$ and $\bar{d}$ is an $m_{i^{\prime}}$-tuple of closed $\mathcal{Q}_{W}$-terms, $\bar{d}=\bar{d}_{m_{a(i)} b(i)}$ for some $i \in \mathbb{N}$, and so the last display provides a $j \in \mathbb{N}$ with

$$
S^{i} \models \exists \bar{y}_{i j} \bigwedge_{k \in \mathbb{N}} \varphi_{i^{\prime} j k}\left(\bar{d}, \bar{y}_{i j}\right) ;
$$

because $S^{i} \subseteq S^{\omega}, \exists \bar{y}_{i j} \bigwedge_{k \in \mathbb{N}} \varphi_{i^{\prime} j k}\left(\bar{d}, \bar{y}_{i j}\right)$ is true in $\mathcal{M}$. Thus it satisfies the given infinitary sentence. As for the complexity of $S^{\omega}$, note that there is a second-order formula $\psi(T, U)$, without set quantifiers, such that $\psi(T, S)$ is satisfied only by $\left\{2^{i} 3^{j}: j \in S^{i}\right\}$ : for $\{0\} \times S^{0}$ may be defined with the help of $\varphi_{0}(S, \mathbb{O}, x)$, and 
each $S^{i+1}$ is obtained from $S^{i}$ just with the help of $F_{2}$ and numerical parameters $S$-recursive in $i$. So $\bigcup_{i \in \mathbb{N}}\{i\} \times S^{i}$ is $\Delta_{1}^{1}(S)$-that is, hyperarithmetical in $S$-as is $S^{\omega}$, since for closed literals $\psi$ from $\mathcal{Q}_{\{0, \ldots, i\}}$,

$$
\psi \in S^{\omega} \quad \text { iff } \quad \psi \in S^{i} .
$$

To apply Theorem 8.3 , one may show that $\mathbb{O}$ forces infinitary sentences, of the kind mentioned in Theorem 8.3, that imply special properties of i.g. dimension groups; for example, being e.c., having no order unit, and having proper self-embeddings as well as automorphisms that are not multiplications by rationals. Corollary 3.6 of [14] implies that the e.c. dimension groups are those models of $T_{\forall}^{\mathfrak{Q}}$ that satisfy the universal closures of all formulas of the following kinds:

(1) $\exists z(x, y<z)$

(2) $x, y<z, v \rightarrow \exists w(x, y<w<z, v)$

(3) $\bigwedge_{\bar{m} \in \mathbb{N}^{n} \backslash\{\overline{0}\}} 0 \not \leq \sum_{i} m_{i} a_{i} \rightarrow \exists y\left(\bigwedge_{i} a_{i}<y \wedge 0 \nless y\right)$

(4) $\bigwedge_{s} \exists y\left(\bigwedge_{i} a_{i}<y \wedge \bigwedge_{j} y<b_{j} \wedge y \mid w_{s}\right) \rightarrow \exists y\left(\bigwedge_{i} a_{i}<y \wedge\right.$ $\left.\bigwedge_{j} y<b_{j} \wedge \bigwedge_{s} y \mid w_{s}\right)$

(5) $\bigwedge_{s} \exists \bar{y}\left(\varphi \wedge y_{s} \mid 0\right) \rightarrow \exists \bar{y}\left(\varphi \wedge \bigwedge_{s} y_{s} \mid 0\right)$ (where $\varphi$ is a conjunction of inequalities in the distinct variables $\bar{y}=\left(y_{s}\right)_{s \in S}$ and $\left.\bar{x}=\left(x_{t}\right)_{t \in T}\right)$

(6) $\bigwedge_{i} a_{i}<0 \wedge \bigwedge_{j} 0<b_{j} \rightarrow \exists y\left(\bigwedge_{i} a_{i}<y \wedge \bigwedge_{j} y<b_{j} \wedge y \mid 0\right)$.

Each of these sentences is forced by $\mathbb{O}$. Consider, for example, $\psi=$

$$
\forall a_{1} \ldots \forall a_{n}\left(\left(\bigwedge_{\bar{m} \in \mathbb{N}^{n} \backslash\{\overline{0}\}} 0 \not 亡 \sum_{i} m_{i} a_{i}\right) \rightarrow \exists y\left(\bigwedge_{i} a_{i}<y \wedge 0 \nless y\right)\right) .
$$

By Theorem 8.1, (10 will force $\psi$ if for distinct witnesses $c_{1}, \ldots, c_{n}$ and any condition $P$ for which $T_{\forall}^{\mathcal{Q}} \cup P \cup\left\{\bigwedge_{\bar{m} \in \mathbb{N}^{n} \backslash\{\overline{0}\}} 0 \not \leq \sum_{i} m_{i} c_{i}\right\}$ is satisfiable, there is a witness $w$ for which $T_{\forall}^{\mathcal{Q}} \cup P \cup\left\{\bigwedge_{\bar{m} \in \mathbb{N}^{n} \backslash\{\overline{0}\}} 0 \not \leq \sum_{i} m_{i} c_{i}, \bigwedge_{i} c_{i}<w \wedge 0 \nless w\right\}$ is satisfiable. This claim holds because every dimension group may be embedded in an e.c. dimension group. One shows in similar fashion that $\mathbb{O}$ forces all the axioms for e.c. dimension groups.

Consider now the sentence

$$
\forall x\left(x>0 \rightarrow \exists y \bigwedge_{n>0} \neg(-n x \leq y \leq n x)\right) .
$$

Theorem 8.1 says that $\mathbb{O}$ forces this sentence just in case for any witness $c$ and any condition $P$ for which $T_{\forall}^{\mathcal{Q}} \cup P \cup\{c>0\}$ is satisfiable, there is a witness $w$ for which $T_{\forall}^{\mathcal{Q}} \cup P \cup\{c>0\} \cup\{\neg(-n c \leq w \leq n c): n>0\}$ is satisfiable; but this claim holds because any dimension group may be embedded in an $\omega$-e.s. dimension group. Thus (1) forces the displayed sentence.

Because the set of $\left(\mathcal{Q}_{F}\right)_{\omega_{1} \omega}$-sentences forced by the $F$-condition $P$ is closed under countable conjunction (see the Appendix), similar arguments handle sentences that guarantee the conclusions of Corollaries 4.4 and 4.9. For each positive integer $k$, let $\theta_{k}\left(x_{1}, \ldots, x_{k}, y_{1}, \ldots, y_{k}\right)$ be the formula

$$
\bigwedge_{\bar{q} \in \mathbb{Q}^{k}}\left[\left(\sum_{i} q_{i} x_{i}<0 \leftrightarrow \sum_{i} q_{i} y_{i}<0\right) \wedge\left(\sum_{i} q_{i} x_{i}=0 \leftrightarrow \sum_{i} q_{i} y_{i}=0\right)\right]
$$


then the sentence relevant to Corollary 4.4 could be

$$
\begin{aligned}
\forall x_{1}\left(x_{1} \neq 0 \rightarrow \exists y_{1}\left(\theta_{1}\left(x_{1}, y_{1}\right) \wedge \bigwedge_{0<q \in \mathbb{Q}} y_{1} \neq q x_{1}\right)\right) \\
\wedge \bigwedge_{k \geq 1} \forall x_{1} \ldots \forall x_{k} \forall x_{k+1} \forall y_{1} \ldots \forall y_{k}\left(\theta_{k}(\bar{x}, \bar{y})\right. \\
\left.\quad \rightarrow \exists y_{k+1} \theta_{k+1}\left(\bar{x}, x_{k+1}, \bar{y}, y_{k+1}\right)\right) \\
\wedge \bigwedge_{k \geq 1} \forall x_{1} \ldots \forall x_{k} \forall y_{1} \ldots \forall y_{k} \forall y_{k+1}\left(\theta_{k}(\bar{x}, \bar{y})\right. \\
\left.\rightarrow \exists x_{k+1} \theta_{k+1}\left(\bar{x}, x_{k+1}, \bar{y}, y_{k+1}\right)\right) .
\end{aligned}
$$

For each positive integer $k$, let $\psi_{k}\left(y_{1}, \ldots, y_{k}, z\right)$ be the formula

$$
\bigwedge_{\bar{q} \in \mathbb{Q}^{k}, r \in \mathbb{Q}}\left(\sum_{i} q_{i} y_{i}=r z \rightarrow r z=0\right) ;
$$

then the sentence relevant to Corollary 4.9 could be

$$
\begin{aligned}
\forall z & \forall x_{1} \exists y_{1}\left(\theta_{1}\left(x_{1}, y_{1}\right) \wedge \psi_{1}\left(y_{1}, z\right)\right) \\
& \wedge \bigwedge_{k \geq 1} \forall z \forall x_{1} \ldots \forall x_{k} \forall x_{k+1} \forall y_{1} \ldots \forall y_{k}\left(\theta_{k}(\bar{x}, \bar{y}) \wedge \psi_{k}(\bar{y}, z)\right. \\
& \left.\rightarrow \exists y_{k+1}\left(\theta_{k+1}\left(\bar{x}, x_{k+1}, \bar{y}, y_{k+1}\right) \wedge \psi_{k+1}\left(\bar{y}, y_{k+1}, z\right)\right)\right) \\
& \wedge \bigwedge_{k \geq 1} \forall z \forall x_{1} \ldots \forall x_{k} \forall y_{1} \ldots \forall y_{k} \forall y_{k+1}\left(\theta_{k}(\bar{x}, \bar{y}) \wedge \psi_{k+1}\left(\bar{y}, y_{k+1}, z\right)\right. \\
& \left.\rightarrow \exists x_{k+1} \theta_{k+1}\left(\bar{x}, x_{k+1}, \bar{y}, y_{k+1}\right)\right) .
\end{aligned}
$$

Given the form of the particular infinitary sentences examined so far, one concludes that their conjunction is logically equivalent to a sentence

$$
\bigwedge_{i \in \mathbb{N}} \forall \bar{x}_{i} \bigvee_{j \in \mathbb{N}} \exists \bar{y}_{i j} \bigwedge_{k \in \mathbb{N}} \varphi_{i j k}\left(\bar{x}_{i}, \bar{y}_{i j}\right),
$$

of the kind subject to Theorem 8.3, that is forced by $\mathbb{O}$ and whose function $(i, j, k) \in \mathbb{N}^{3} \mapsto \forall \bar{x}_{i} \exists \bar{y}_{i j} \varphi_{i j k}\left(\bar{x}_{i}, \bar{y}_{i j}\right)$ is recursive. So Theorem 8.3 implies the next result.

Corollary 8.4 There is a hyperarithmetic e.c. dimension group $\mathcal{E}$, without an order unit, in which every $a \neq 0$ is automorphic to some element of $G \backslash\{q a$ : $0<q \in \mathbb{Q}\}$ and fails to belong to the image of some self-embedding of $\mathcal{G}$.

Because no i.g. dimension group is analytical (see Corollary 6.5), the algebraic properties, discovered so far, that seem peculiar to i.g. dimension groups do not characterize them. When treating an analogous result for f.g. dimension groups, Section 7 pointed out that the class of f.g. dimension groups is axiomatized by a sentence of the kind subject to Theorem 7.4, and that analysis of the complexity of this sentence allowed one to find an f.g. dimension group recursive in first-order arithmetic. No similar strategy will reveal an i.g. dimension group recursive in second-order arithmetic because of the following result.

Lemma 8.5 No sentence of $\mathcal{Q}_{\omega_{1} \omega}$ axiomatizes the class of i.g. dimension groups. 
Proof Suppose that the sentence $\varphi$ of $\mathcal{Q}_{\omega_{1} \omega}$ does axiomatize the class of i.g. dimension groups. If $c$ is a new constant symbol, the sentence $\varphi \wedge 0<c$ axiomatizes the class of i.g. dimension groups with distinguished positive element. This class of structures is the same as the class of infinitely generic models of $T^{\mathcal{Q}} \cup\{0<c\}=T^{\prime}$.

Section 5 provides a first-order $\mathcal{Q} \cup\{c\}$-formula $N(x)=$

$$
x \in \mathbb{N} \cdot c
$$

with $N^{\mathcal{M}}=\mathbb{N} \cdot c^{\mathcal{M}}$ for all e.c. dimension groups $\mathcal{M}$ with $0<c^{\mathcal{M}}$.

Section 6 provides a first-order $\mathcal{Q} \cup\{c\}$-formula $S(x, y)=$

$$
\mu(c, y, x)
$$

with the following property: if $\mathcal{M}$ is an e.c. dimension group with $0<c^{\mathcal{M}}$, $X \subseteq \mathbb{N} \cdot c^{\mathcal{M}}$, and $\mathcal{N} \supseteq \mathcal{M}$ is an $\omega$-e.s. dimension group, then there is $e \in N$ with

$$
X=\left\{d \in \mathbb{N} \cdot c^{\mathcal{M}}: \mathcal{N} \models S(d, e)\right\} .
$$

If $\kappa(y, z, w)$ is an unnested formula, in the language of first-order arithmetic, that defines $\left\{\left(a, b, 2^{a} 3^{b}\right): a, b \in \mathbb{N}\right\}$ and does not contain the variable $x$, Lemma 6.3 implies that in any e.c. dimension group $\mathcal{M}$ with $0<c^{\mathcal{M}}, \kappa^{(c)}(y, z, w)$ defines

$$
\left\{\left(a c^{\mathcal{M}}, b c^{\mathcal{M}}, 2^{a} 3^{b} c^{\mathcal{M}}\right): a, b \in \mathbb{N}\right\} .
$$

Let $R\left(x^{\prime}, y, z\right)$ be the $(\mathcal{Q} \cup\{c\})$-formula $\left(\exists w\left(\kappa\left(x^{\prime}, y, w\right) \wedge w \in Z\right)\right)^{(c)}$; that is, $\exists w \in \mathbb{N} \cdot c\left(\kappa^{(c)}\left(x^{\prime}, y, w\right) \wedge \mu(c, z, w)\right)$. If $\mathcal{M} \models \varphi \wedge 0<c, \Lambda$ is a linear ordering of $\mathbb{N} \cdot c^{\mathcal{M}}$, and $\mathcal{N} \supseteq \mathcal{M}$ is an $\omega$-e.s. dimension group, then there is $e \in N$ with

$$
\Lambda=\left\{(a, b) \in\left(\mathbb{N} \cdot c^{\mathcal{M}}\right)^{2}: \mathcal{N} \models R(a, b, e)\right\} .
$$

Because the formulas $N, R, S$ have these properties, [9, Theorem 1] implies that no sentence of $(\mathcal{Q} \cup\{c\})_{\omega_{1} \omega}$ axiomatizes the class of i.g. models of $T^{\prime}$, and one reaches a contradiction.

But a slight modification of Lemma 8.5 yields more positive conclusions. First, one may state the modification.

Lemma 8.6 There is no sentence of $\mathcal{Q}_{\omega_{1} \omega}$ whose countable models are exactly the countable i.g. dimension groups.

Proof Suppose otherwise, and let $\varphi$ be such a sentence. If $c$ is a new constant symbol, the countable models of $\varphi \wedge 0<c$ are the countable i.g. models of the theory $T^{\prime}=T^{\mathcal{Q}} \cup\{0<c\}$. Let $N, R$, and $S$ be the formulas from the proof of Lemma 8.5.

$N^{\mathcal{M}}=\mathbb{N} \cdot c^{\mathcal{M}}$ for all countable $\mathcal{M} \models \varphi \wedge 0<c$.

If $\mathcal{M} \models \varphi \wedge 0<c$ is countable, $X \subseteq \mathbb{N} \cdot c^{\mathcal{M}}$, and $\mathcal{N} \supseteq \mathcal{M}$ is an $\omega$-e.s. dimension group, then there is $e \in N$ with

$$
X=\left\{d \in \mathbb{N} \cdot c^{\mathcal{M}}: \mathcal{N} \models S(d, e)\right\} .
$$

If $\mathcal{N}^{\prime} \preccurlyeq \mathcal{N}$ is countable with $M \cup\{e\} \subseteq N^{\prime}$, then $\mathcal{N}^{\prime}$ is a countable i.g. model of $T^{\prime}$ and

$$
X=\left\{d \in \mathbb{N} \cdot c^{\mathcal{N}^{\prime}}: \mathcal{N}^{\prime} \models S(d, e)\right\}
$$


A similar argument shows that if $\mathcal{M} \models \varphi \wedge 0<c$ is countable and $\Lambda$ is a linear ordering of $\mathbb{N} \cdot c^{\mathcal{M}}$, then there are a countable extension $\mathcal{N} \models \varphi \wedge 0<c$ of $\mathcal{M}$ and $e \in N$ with

$$
\Lambda=\left\{(a, b) \in\left(\mathbb{N} \cdot c^{\mathcal{M}}\right)^{2}: \mathcal{N} \models R(a, b, e)\right\} .
$$

Because the formulas $N, R, S$ have these properties, one may follow the steps in the proof of [9, Theorem 1] without using uncountable models. One thus obtains a contradiction showing that $\varphi$ does not exist.

With the help of Lemma 8.6, one reaches the following conclusion.

Theorem 8.7 There is a countable e.c. dimension group $\mathcal{E}$ that satisfies $T^{F}$, is not i.g., has no order unit, and in which every $a \neq 0$ is automorphic to some element of $G \backslash\{q a: 0<q \in \mathbb{Q}\}$ and fails to belong to the image of some self-embedding of $\mathcal{E}$.

Proof The conjunction $\varphi$ of the sentences in $T^{\mathrm{F}}$ with the infinitary sentence exploited in the proof of Corollary 8.4 is a sentence of $\mathcal{Q}_{\omega_{1} \omega}$ true in all i.g. dimension groups; so by Lemma 8.6 there is a countable model $\mathcal{G}$ of $\varphi$ that is not i.g. Since $\varphi \models T^{\mathrm{F}}, \mathcal{E} \models T^{\mathrm{F}}$; since $\varphi$ implies the axioms for e.c. dimension groups, $\mathscr{E}$ is an e.c. dimension group; and because $\mathcal{E}$ is countable, the other infinitary conjuncts of $\varphi$ give $\mathcal{G}$ the remaining special properties mentioned above.

This structure cannot be hyperarithmetic because a hyperarithmetic structure has a hyperarithmetic first-order theory.

While Lemma 8.5 rules out the first strategy for proving an i.g. version of Corollary 7.5 , the second strategy is still available. I.g. structures were originally defined to be $\mathcal{Q}$-structures $\mathcal{M}$ in which

$$
\mathcal{M} \Vdash \varphi \quad \text { or } \quad \mathcal{M} \Vdash \neg \varphi \quad \text { for all } \mathcal{Q}_{M} \text {-sentences } \varphi
$$

with connectives and quantifiers among $\wedge, \vee, \neg$, and $\exists$. The definition of $\|$, a relation between $\mathcal{Q}$-structures $\mathcal{M} \models T_{\forall}^{\mathcal{Q}}$ and $\mathcal{Q}_{M}$-sentences $\varphi$ as above, went as follows (see [6, p. 56]). When $\varphi$ is atomic,

$$
\begin{array}{rll}
\mathcal{M} \| \varphi & \text { iff } & \mathcal{M} \models \varphi ; \\
\mathcal{M} \models \theta \vee \gamma & \text { iff } & \mathcal{M} \models \theta \text { or } \mathcal{M} \models \gamma ; \\
\mathcal{M} \models \theta \wedge \gamma & \text { iff } & \mathcal{M} \models \theta \text { and } \mathcal{M} \models \gamma ; \\
\mathcal{M} \| \exists x \psi(x) & \text { iff } & \text { for some } a \in M, \mathcal{M} \| \psi(a) ;
\end{array}
$$

and

$$
\mathcal{M} \| \neg \theta \quad \text { iff } \quad \text { for all } \mathcal{N} \supseteq \mathcal{M} \text { with } \mathcal{N} \models T_{\forall}^{\mathcal{Q}}, \mathcal{N} \nVdash=\theta \text {. }
$$

Because the structures $\mathcal{M}$ may be uncountable, one cannot treat $\|$ directly in second-order arithmetic. But an analogous relation $\|$, between $F$-conditions $P$ and sentences $\varphi$ of $\mathcal{Q}_{F}$, is much more closely related to second-order arithmetic. When $\varphi$ is atomic,

$$
\begin{array}{rll}
P \| \varphi & \text { iff } & \varphi \in P ; \\
P \| \theta \vee \gamma & \text { iff } & P \| \theta \text { or } P \| \gamma ; \\
P \| \theta \wedge \gamma & \text { iff } & P \| \theta \text { and } P \| \gamma ; \\
P \| \exists x \psi(x) & \text { iff } & \text { there is a closed } Q_{F} \text {-term } t \text { with } P \| \psi(t) ;
\end{array}
$$


and

$$
P \| \neg \theta \quad \text { iff } \quad \text { for all conditions } Q \supseteq P, Q \nVdash \theta \text {. }
$$

When $P$ is an $F$-condition, let $\mathcal{M}_{P}$ be the $\mathcal{Q}$-reduct of the $\mathcal{Q}_{F}$-structure with diagram $P$. The following result relates these two notions of forcing.

Lemma 8.8 If $\varphi\left(x_{1}, \ldots, x_{n}\right)$ is a $\mathcal{Q}_{\text {-formula, }} t_{1}, \ldots, t_{n}$ are closed $\mathcal{Q}_{F}$-terms, and $t_{i}^{\mathcal{M}_{P}}=a_{i}$ for $i=1, \ldots, n$, then

$$
P \| \varphi(\bar{t}) \quad \text { iff } \quad \mathcal{M}_{P} \| \varphi(\bar{a}) .
$$

Proof The argument goes by induction on the complexity of $\varphi(\bar{x})$, and the only case demanding discussion is the induction step for $\neg$.

Suppose that $\mathcal{M}_{P} \| \neg \varphi(\bar{a})$ and that $R$ is a condition extending $P$; then $\mathcal{M}_{R} \supseteq \mathcal{M}_{P}$, and so by hypothesis $\mathcal{M}_{R} \nVdash \models \varphi(\bar{a})$ and by induction hypothesis $R \nVdash \models \varphi(\bar{t})$. Thus $P \| \neg \varphi(\bar{t})$ if $\mathcal{M}_{P} \| \neg \varphi(\bar{a})$.

Assume now that $P \| \neg \varphi(\bar{t})$. If $\mathcal{M}_{P} \nvdash \neg \neg \varphi(\bar{a})$, then there is a model $\mathcal{N} \supseteq \mathcal{M}$ of $T_{\forall}^{\mathcal{Q}}$ with $\mathcal{N} \Vdash \varphi(\bar{a})$. The Löwenheim-Skolem theorem provides an at most countable $\mathcal{K} \preccurlyeq \mathcal{N}$ with $M_{P} \cup\left\{a_{1}, \ldots, a_{n}\right\} \subseteq K$, and by Robinson's reduction theorem (see [6, Theorem 3.13])

$$
\mathcal{K} \| \varphi(\bar{a}) .
$$

Since $K \supseteq M_{P}$ is at most countable, one may view $\mathcal{K}$ as $\mathcal{M}_{Q}$ for some condition $Q \supseteq P$, and by induction hypothesis $Q \Vdash \varphi(\bar{t})$, though $P \| \neg \varphi(\bar{t})$. This contradiction shows that $\mathcal{M}_{P} \| \neg \varphi(\bar{a})$ if $P \| \neg \varphi(\bar{t})$.

One may now sketch the proof of the following result.

Lemma 8.9 There is a recursive sequence $\left\{\varphi_{n}^{2}(X, y)\right\}_{n \geq 0}$, of formulas of secondorder arithmetic, with the following property: when $P$ is an $F$-condition, $\varphi_{n}^{2}(P, y)$ defines the set of all $Q_{F}$-sentences $\varphi$, of complexity at most $n$, with $P \| \varphi$.

Proof If $C(X, y)$ is the $\Pi_{1}^{0}$-formula defining the set of all pairs $(A,\ulcorner F\urcorner)$ such that $A$ is an $F$-condition, then $\exists y C(X, y)\left(=C^{\prime}(X)\right)$ defines the set of all conditions. One may let $\varphi_{0}^{2}(X, y)$ be a translation of

$$
C^{\prime}(X) \wedge y \in X \wedge \text { " } y \text { is an atomic } \mathcal{Q}_{W} \text {-sentence." }
$$

Given $\varphi_{n}^{2}(X, y)$, one may let $\varphi_{n+1}^{2}(X, y)$ be a translation of

$$
\begin{aligned}
\left(" y \text { is a } \mathcal{Q}_{W} \text {-sentence of complexity } \leq n " \wedge \varphi_{n}^{2}(X, y)\right) \\
\vee\left[" y \text { is a } \mathcal{Q}_{W} \text {-sentence of complexity } n+1 "\right. \\
\wedge\left\{\left(" y \text { is a disjunction } \theta \vee \gamma " \wedge\left[\varphi_{n}^{2}(X, \theta) \vee \varphi_{n}^{2}(X, \gamma)\right]\right)\right. \\
\vee\left(" y \text { is a conjunction } \theta \wedge \gamma " \wedge\left[\varphi_{n}^{2}(X, \theta) \wedge \varphi_{n}^{2}(X, \gamma)\right]\right) \\
\vee(" y \text { is an existential quantification } \exists x \varphi(x) " \\
\left.\wedge \exists t\left(t \text { is a closed instance of } y \wedge \varphi_{n}^{2}(X, t)\right)\right) \\
\vee(" y \text { is a negation } \neg \theta " \\
\left.\left.\left.\wedge \forall Z\left(X \subseteq Z \wedge C^{\prime}(Z) \rightarrow \neg \varphi_{n}^{2}(Z, \theta)\right)\right)\right\}\right] .
\end{aligned}
$$

These results allow one to prove an analogue of Corollary 7.5. 
Theorem 8.10 Assume that there is an analytical well-ordering of the power set of the natural numbers, ${ }^{12}$ then there is an i.g. dimension group recursive in secondorder arithmetic.

Proof Let $\left\{\theta_{n}\right\}_{n \in \mathbb{N}}$ be a recursive enumeration of all $\mathcal{Q}_{W}$-sentences-with $\theta_{2 n}$ equal to $c_{n, 0}=c_{n, 0}$ always-and let $d: \mathbb{N} \rightarrow \mathbb{N}$ be a recursive function such that for all $n \in \mathbb{N}, d(n)$ is an upper bound on the complexity of $\theta_{0}, \ldots, \theta_{n}$. Let $X \prec Y$ be a formula, of second-order arithmetic, defining a well-ordering of the power set of the natural numbers. There is a recursive sequence $\left\{\delta_{n}(x)\right\}_{n \in \mathbb{N}}$, of formulas of second-order arithmetic, that defines an increasing chain of conditions $S^{n}$, where

$$
S^{n} \Vdash \theta_{n} \quad \text { or } \quad S^{n} \Vdash \neg \theta_{n} \quad \text { always; }
$$

and at each stage one chooses the least condition that does the job. Thus $\delta_{0}(x)$ may be

$$
\begin{aligned}
\forall V & {\left[\left\{\left(\varphi_{d(0)}^{2}\left(V, \theta_{0}\right) \vee \varphi_{d(0)}^{2}\left(V, \neg \theta_{0}\right)\right)\right.\right.} \\
& \left.\left.\wedge \forall V^{\prime}\left(\left(\varphi_{d(0)}^{2}\left(V^{\prime}, \theta_{0}\right) \vee \varphi_{d(0)}^{2}\left(V^{\prime}, \neg \theta_{0}\right)\right) \rightarrow V \preccurlyeq V^{\prime}\right)\right\} \rightarrow x \in V\right]
\end{aligned}
$$

and $\delta_{n+1}(x)$ may be

$$
\begin{aligned}
\forall V & {\left[\left\{\left(\varphi_{d(n+1)}^{2}\left(V, \theta_{n+1}\right) \vee \varphi_{d(n+1)}^{2}\left(V, \neg \theta_{n+1}\right)\right) \wedge \forall x\left(\delta_{n}(x) \rightarrow x \in V\right)\right.\right.} \\
& \wedge \forall V^{\prime}\left(\left(\varphi_{d(n+1)}^{2}\left(V^{\prime}, \theta_{n+1}\right) \vee \varphi_{d(n+1)}^{2}\left(V^{\prime}, \neg \theta_{n+1}\right)\right) \wedge \forall x\left(\delta_{n}(x) \rightarrow x \in V^{\prime}\right)\right. \\
& \left.\left.\rightarrow V \preccurlyeq V^{\prime}\right)\right\} \\
& \rightarrow x \in V] .
\end{aligned}
$$

Because $\theta_{2 n}$ is $c_{n, 0}=c_{n, 0}$ for all $n \in \mathbb{N}, S^{2 n}$ is always an $F$-condition for some $F \supseteq\{0, \ldots, n\}$, and $S^{\omega}=\bigcup_{n} S^{n}$ is the diagram of a $\mathcal{Q}$-structure $\mathcal{M} \models T_{\forall}^{\mathcal{Q}}$. Also, a literal $\theta$ of $\mathcal{Q}_{\{0, \ldots, n\}}$ belongs to $S^{\omega}$ just in case $\theta$ belongs to $S^{2 n}$; that is, just in case $\delta_{2 n}(\theta)$ is true in second-order arithmetic. Thus $S^{\omega}$ is recursive in second-order arithmetic. Suppose that $\psi\left(x_{1}, \ldots, x_{n}\right)$ is a $\mathcal{Q}$-formula and that $a_{1}, \ldots, a_{n} \in M$. There are closed $\mathcal{Q}_{W}$-terms $t_{1}, \ldots, t_{n}$ with $a_{i}=t_{i}^{\mathcal{M}}$ for $i=1, \ldots, n$, and $\psi\left(t_{1}, \ldots, t_{n}\right)$ is $\theta_{n}$ for some $n \in \mathbb{N}$. If $S^{n} \Vdash \theta_{n}$, then by Lemma 8.8

$$
\mathcal{M}_{S^{n}} \| \psi(\bar{a}),
$$

and so $\mathcal{M} \Vdash \psi(\bar{a})$ since $\mathcal{M}_{S^{n}} \subseteq \mathcal{M}$; a similar argument shows that $\mathcal{M} \| \neg \psi(\bar{a})$ if $S^{n} \| \neg \theta_{n}$. Thus

$$
\mathcal{M} \| \gamma \quad \text { or } \quad \mathcal{M} \Vdash \neg \gamma \quad \text { for all } \mathcal{Q}_{M} \text {-sentences } \gamma
$$

and $\mathcal{M}$ is i.g.

\section{Some Open Questions}

Are there mathematically natural characterizations of the generic dimension groups? All one can say now is that the currently known special algebraic properties of these groups are too weak because they may be expressed in the forms handled by Theorems $7.4,8.3$, and 8.7 .

Do the numbers $\alpha<\beta$ provide isomorphism invariants for f.g. dimension groups? Suppose that $\mathscr{D}$ and $\mathscr{D}^{\prime}$ are f.g. dimension groups, $a \in D$ and $a^{\prime} \in D^{\prime}$ are positive, 
and

$$
\begin{aligned}
& \left\{(\alpha, \beta) \in \mathbb{R}^{2}: \exists b \in D \backslash \mathbb{Q} \cdot a \forall t \in \mathbb{Q}\right. \\
& \quad[(t<\alpha \text { iff } t a<b) \text { and }(\alpha<t<\beta \text { iff } t a \mid b) \text { and }(\beta<t \text { iff } b<t a)]\}
\end{aligned}
$$

equals

$$
\begin{aligned}
& \left\{(\alpha, \beta) \in \mathbb{R}^{2}: \exists b^{\prime} \in D^{\prime} \backslash \mathbb{Q} \cdot a^{\prime} \forall t \in \mathbb{Q}\right. \\
& \left.\quad\left[\left(t<\alpha \text { iff } t a^{\prime}<b^{\prime}\right) \text { and }\left(\alpha<t<\beta \text { iff } t a^{\prime} \mid b^{\prime}\right) \text { and }\left(\beta<t \text { iff } b^{\prime}<t a^{\prime}\right)\right]\right\} .
\end{aligned}
$$

Is $(\mathscr{D}, a) \cong\left(\mathscr{D}^{\prime}, a^{\prime}\right)$ ?

May one explain the existence of uncountable f.g. dimension groups (see [14, Theorem 6.28]) by a joint-embedding property for f.g. dimension groups? A positive answer to this question could yield a positive answer to the question about the numbers $\alpha<\beta$; for suppose that the groups $(\mathscr{D}, a)$ and $\left(\mathscr{D}^{\prime}, a^{\prime}\right)$ of the last paragraph may be embedded into $(\mathscr{H}, h)$, where $\mathscr{G}$ is f.g. and $(\mathscr{D}, a),\left(\mathscr{D}^{\prime}, a^{\prime}\right)$, and $(\mathscr{E}, h)$ are $(\mathcal{Q} \cup\{u\})$-structures with $u$ a new constant symbol. If $f:(\mathscr{D}, a) \rightarrow(\mathscr{E}, h)$ and $f^{\prime}:\left(\mathscr{D}^{\prime}, a^{\prime}\right) \rightarrow(\mathcal{E}, h)$ are $\mathcal{Q} \cup\{u\}$-embeddings, Theorem 2.3 may be applied to $x=h$ to show that $\operatorname{ran}(f)=\operatorname{ran}\left(f^{\prime}\right):$ so $(\mathscr{D}, a) \cong\left(\mathscr{D}^{\prime}, a^{\prime}\right)$ via $\left(f^{\prime}\right)^{-1} \circ f$.

Is there a recursive e.c. dimension group $\mathcal{E}$, with no order unit, in which every $a \neq 0$ is automorphic to some $b \in G \backslash\{q a: 0<q \in \mathbb{Q}\}$ and fails to belong to the image of some self-embedding of $\mathscr{E}$ ?

If $U \subseteq T^{\mathrm{f}}$ and $\emptyset$ forces

$$
\bigwedge_{i \in \mathbb{N}} \forall \bar{x}_{i} \bigvee_{j \in \mathbb{N}} \exists \bar{y}_{i j} \varphi_{i j}\left(\bar{x}_{i}, \bar{y}_{i j}\right)
$$

(a sentence as in Lemma 7.3), is there a $\mathcal{Q}$-structure, satisfying this infinitary sentence and the sentences in $U$, that is recursive in the join of $U$ with $(i, j) \mapsto \forall \bar{x}_{i} \exists \bar{y}_{i j} \varphi_{i j}\left(\bar{x}_{i}, \bar{y}_{i j}\right)$ ? If $U \subseteq T^{\mathrm{F}}$ and $\mathbb{O}$ forces

$$
\bigwedge_{i \in \mathbb{N}} \forall \bar{x}_{i} \bigvee_{j \in \mathbb{N}} \exists \bar{y}_{i j} \bigwedge_{k \in \mathbb{N}} \varphi_{i j k}\left(\bar{x}_{i}, \bar{y}_{i j}\right)
$$

(a sentence as in Lemma 8.2), is there a $\mathcal{Q}$-structure, satisfying this infinitary sentence and the sentences in $U$, that is hyperarithmetic in the join of $U$ with $(i, j, k) \in \mathbb{N}^{3} \mapsto \forall \bar{x}_{i} \exists \bar{y}_{i j} \varphi_{i j k}\left(\bar{x}_{i}, \bar{y}_{i j}\right)$ ?

Does the machinery behind Theorems 7.4 and 8.3 produce generic abelian $l$-groups with interesting properties? This machinery relies on no properties of $T^{\mathcal{Q}}$ that are not shared by the first-order theory of divisible abelian $l$-groups in the language $\{+,-, \wedge, \vee, 0\} \cup\{q \cdot: q \in \mathbb{Q}\}$.

Finally, does ZFC imply the existence of an i.g. dimension group recursive in second-order arithmetic?

\section{Appendix}

This Appendix establishes relations, between different notions of model-theoretic forcing, exploited in the arguments of earlier sections.

Hirschfeld and Wheeler define a relation

$$
p \Vdash_{\mathrm{HW}} \varphi,
$$


between conditions $p$ (for finite forcing relative to $T^{\mathcal{Q}}$ ) and $\mathcal{Q}_{W}$-sentences with connectives and quantifiers among $\wedge, \vee, \neg$, and $\exists$ (see [6, p. 87]), while Hodges defines a relation

$$
p \text { forces } \varphi
$$

between conditions $p$ and $\mathcal{Q}_{W}$-sentences $\varphi$ (see [7, Section 3.4]). As in [6, p. 89], write

$$
p \Vdash_{\mathrm{HW}}^{*} \varphi
$$

for

$$
p \Vdash_{\mathrm{HW}} \neg \neg \varphi .
$$

Lemma A.1 For $\mathcal{Q}_{W}$-sentences $\varphi$ with connectives and quantifiers among $\wedge, \vee, \neg$, and $\exists$,

$$
p \Vdash_{\mathrm{HW}}^{*} \varphi \quad \text { iff } \quad \text { p forces } \varphi .
$$

Proof The argument goes by induction on the complexity of $\varphi$.

If $\varphi$ is atomic, then by [6, Proposition 5.7]

$$
\begin{array}{rll}
p \Vdash_{\mathrm{HW}}^{*} \varphi & \text { iff } & T^{\mathcal{Q}} \cup p \models \neg \neg \varphi \\
& \text { iff } & T^{\mathcal{Q}} \cup p \models \varphi,
\end{array}
$$

and so

$$
p \Vdash_{\mathrm{HW}}^{*} \varphi \quad \text { iff } \quad p \text { forces } \varphi
$$

by [7, Theorem 3.4.2].

Assume that the $\mathcal{Q}_{W}$-sentences $\varphi$ and $\psi$ obey the result. By [6, Proposition 5.5],

$$
\begin{array}{rll}
p \Vdash_{\mathrm{HW}}^{*} \varphi \wedge \psi & \text { iff } & p \Vdash_{\mathrm{HW}}^{*} \varphi \text { and } p \Vdash_{\mathrm{HW}}^{*} \psi \\
& \text { iff } & p \text { forces } \varphi \text { and } p \text { forces } \psi \quad \text { (by induction hypothesis) } \\
& \text { iff } & p \text { forces } \varphi \wedge \psi \quad([7, \text { Theorem } 2.3 .4(\mathrm{~d})]) .
\end{array}
$$

Also,

$$
\begin{aligned}
& p \Vdash_{\mathrm{HW}}^{*} \varphi \vee \psi \quad \text { iff } \quad p \Vdash_{\mathrm{HW}}^{*} \neg \neg(\varphi \vee \psi) \\
& \text { iff for every condition } q \supseteq p \text { there is a condition } r \supseteq q \\
& \text { with } r \Vdash_{\text {HW }} \varphi \text { or } r \Vdash_{\text {HW }} \psi \\
& \Rightarrow \quad \text { for every condition } q \supseteq p \text { there is a condition } r \supseteq q \\
& \text { with } r \Vdash_{\mathrm{HW}}^{*} \varphi \text { or } r \Vdash_{\mathrm{HW}}^{*} \psi \quad([6, \text { Lemma 5.4(iii)]) } \\
& \Rightarrow \quad \text { for every condition } q \supseteq p \text { there is a condition } r \supseteq q \\
& \text { that forces } \varphi \text { or forces } \psi \text { (induction hypothesis) } \\
& \Rightarrow \quad \text { for every condition } q \supseteq p \text { there is a condition } r \supseteq q \\
& \text { that forces } \varphi \vee \psi \quad([7 \text {, Theorem 2.3.4(b)]) } \\
& \Rightarrow \quad p \text { forces } \varphi \vee \psi \quad([7, \text { Exercise 3.4.1(a)]) }
\end{aligned}
$$

and

$$
\begin{array}{ll}
p \|_{\mathrm{HW}}^{*} \varphi \vee \psi & \\
\text { iff } & p \nvdash_{\mathrm{HW}}^{*}(\neg \neg \varphi) \vee(\neg \neg \psi) \quad([6, \text { Proposition 5.5]) } \\
\text { iff } \quad \text { there is a condition } q \supseteq p \text { such that for all conditions } r \supseteq q, \\
& \quad r \nvdash_{\mathrm{HW}}(\neg \neg \varphi) \vee(\neg \neg \psi)
\end{array}
$$


iff there is a condition $q \supseteq p$ such that for all conditions $r \supseteq q$, $r \|{ }_{\text {HW }} \neg \neg \varphi$ and $r \nvdash_{\text {HW }} \neg \neg \psi$

iff there is a condition $q \supseteq p$ such that for all conditions $r \supseteq q$, $r \forall_{\mathrm{HW}}^{*} \varphi$ and $r \nvdash_{\mathrm{HW}}^{*} \psi$

iff there is a condition $q \supseteq p$ such that for all conditions $r \supseteq q$, $r$ forces neither $\varphi$ nor $\psi \quad$ (induction hypothesis)

iff $\quad$ there is a condition $q \supseteq p$ forcing both $\neg \varphi$ and $\neg \psi$

([7, Theorem 2.3.4(f)])

$\Rightarrow \quad p$ does not force $\varphi \vee \psi$.

And by the definition of $\vdash_{\mathrm{HW}}^{*}$,

$$
\begin{array}{rll}
p \Vdash_{\mathrm{HW}}^{*} \neg \varphi \quad \text { iff } & \text { for all conditions } q \supseteq p, q \nvdash_{\mathrm{HW}} \neg \neg \varphi \\
\text { iff } & \text { for all conditions } q \supseteq p, q \forall_{\mathrm{HW}}^{*} \varphi \\
\text { iff } & \text { for all conditions } q \supseteq p, q \text { does not force } p \\
& \text { (induction hypothesis) } \\
\text { iff } & p \text { forces } \neg \varphi \quad([7, \text { Theorem 2.3.4(f)]). }
\end{array}
$$

Suppose finally that $\varphi(x)$ is a $\mathcal{Q}_{W}$-formula, whose connectives and quantifiers are among $\wedge, \vee, \neg$, and $\exists$, such that every closed instance of $\varphi(x)$ obeys the result; then $p \Vdash_{\mathrm{HW}}^{*} \exists x \varphi(x) \quad$ iff $\quad$ for every condition $q \supseteq p$ there is a condition $r \supseteq q$ with $r \Vdash_{\text {HW }} \exists x \varphi(x)$

iff $\quad$ for every condition $q \supseteq p$ there are a condition $r \supseteq q$ and a closed $\mathcal{Q}_{W}$-term $t$ with $r \Vdash_{\text {HW }} \varphi(t)$

$\Rightarrow \quad$ for every condition $q \supseteq p$ there are a condition $r \supseteq q$ and a closed $\mathcal{Q}_{W}$-term $t$ with $r \Vdash_{\mathrm{HW}}^{*} \varphi(t)$

([6, Lemma 5.4(iii)])

$\Rightarrow \quad$ for every condition $q \supseteq p$ there are a condition $r \supseteq q$ and a closed $\mathcal{Q}_{W}$-term $t$ with $r$ forcing $\varphi(t)$

(induction hypothesis)

$\Rightarrow \quad$ for every condition $q \supseteq p$ there are a condition $s \supseteq q$ and a witness $c$ with $s$ forcing $\varphi(c)$ (let $c$ be a witness not in $r, t$, or $\varphi(t)$ and let $s=r \cup\{t=c\})$

$\Rightarrow \quad p$ forces $\exists x \varphi(x) \quad$ ([7, Theorem 3.4.1(a)]).

Conversely,

$p \nvdash_{\mathrm{HW}}^{*} \exists x \varphi(x) \quad$ iff $\quad p \nvdash_{\mathrm{HW}}^{*} \exists x \neg \neg \varphi(x) \quad$ ([6, Proposition 5.5])

iff there is a condition $q \supseteq p$ such that for all conditions $r \supseteq q, r \forall{ }_{\text {HW }} \exists x \neg \neg \varphi(x)$

iff there is a condition $q \supseteq p$ such that for all conditions $r \supseteq q$ and closed $\mathcal{Q}_{W}$-terms $t, r \forall_{\mathrm{HW}} \neg \neg \varphi(t)$ 
iff there is a condition $q \supseteq p$ such that for all conditions $r \supseteq q$ and closed $\mathcal{Q}_{W}$-terms $t, r$ does not force $\varphi(t)$ (induction hypothesis)

$\Rightarrow \quad$ there is a condition $q \supseteq p$ such that for all conditions $r \supseteq q$ and witnesses $c, r$ does not force $\varphi(c)$ (induction hypothesis)

$\Rightarrow \quad p$ does not force $\exists x \varphi(x) \quad$ ([7, Exercise 3.4.1(a)]).

An immediate corollary runs as follows.

Corollary A.2 The finite-forcing companion of [7] is the same as the finite-forcing companion of [6].

Proof The finite-forcing companion of [7, p. 77] is the set of $\mathcal{Q}$-sentences forced by $\emptyset$, while the finite-forcing companion of $[6$, p. 89] is the set of $\mathcal{Q}$-sentences $\varphi$, whose connectives and quantifiers are among $\wedge, \vee, \neg$, and $\exists$, such that $\varnothing \Vdash_{\text {HW }}^{*} \varphi$. Since when dealing with first-order theories one may assume that all sentences have connectives and quantifiers among $\wedge, \vee, \neg$, and $\exists$, the desired conclusion follows from Lemma A.1.

Turning now from finite forcing to the forcing, with $\mathcal{Q}_{F}$-diagrams as conditions, introduced at the start of Section 8, one may point out properties that this forcing relation shares with the relations discussed in [7, Sections 2.1, 2.3].

In (1) of [7, p. 19], " $p \cup\{t=t\} \in N$ " should be replaced by: $p \cup\{t=t\}$ is contained in a condition.

In (2) of [7, p. 19], $p \cup\{\varphi(t)\}$ will equal $p$.

In (1) of [7, p. 27], " $p \cup\{t=c\} \in N$ " should be replaced by: $p \cup\{t=c\}$ is contained in a condition.

(2) of [7, p. 27] should be replaced by: witnesses from at most finitely many levels occur in any given condition.

Lemmas 2.3.1-2.3.3 and Theorem 2.3.4 continue to hold, and by the same proofs. The analogue of Theorem 2.3.4 immediately implies the next result.

Corollary A.3 If $\psi$ is the $\left(\mathcal{Q}_{W}\right)_{\omega_{1} \omega^{- \text {sentence }}}$

$$
\forall \bar{x} \bigvee_{i \in \mathbb{N}} \exists \bar{y}_{i} \varphi_{i}\left(\bar{x}, \bar{y}_{i}\right)
$$

and $P$ is a condition, then $P$ forces $\psi$ just in case for all witnesses $\bar{c}$ and conditions $Q \supseteq P$ there are $i \in \mathbb{N}$, witnesses $\bar{d}_{i}$, and a condition $R \supseteq Q$ that forces $\varphi_{i}\left(\bar{c}, \bar{d}_{i}\right)$.

Quantifier-free sentences are forced under the following circumstances.

Lemma A.4 Suppose that $\theta$ is a quantifier-free sentence of $\left(\mathcal{Q}_{F}\right)_{\omega_{1} \omega}$ and that $P$ is an F-condition; then

$$
P \text { forces } \theta \quad \text { iff } \quad P \models \theta \text {. }
$$

Proof The argument goes by induction on the complexity of $\theta$, whose connectives may be restricted to $\neg$ and countable conjunction.

Suppose that $\theta$ is atomic. If $P \models \theta$, then $\theta \in P$ because $P$ is an $F$-condition, and so $P$ forces $\theta$ by the analogue of Theorem 2.3.4(c). If $P \forall \theta$, then $(\neg \theta) \in P$ 
because $P$ is an $F$-condition; so $\theta \notin Q$ for all conditions $Q \supseteq P, P$ forces $\neg \theta$ by the analogue of Theorem 2.3.4(f), and so $P$ does not force $\theta$.

If $\theta$ is $\bigwedge_{i \in \mathbb{N}} \theta_{i}$, where each $\theta_{i}$ is a quantifier-free sentence of $\left(\mathcal{Q}_{F}\right)_{\omega_{1} \omega}$ that obeys the result, then $\theta$ obeys it by the analogue of Theorem 2.3.4(d).

Suppose now that the quantifier-free $\left(\mathcal{Q}_{F}\right)_{\omega_{1} \omega}$-sentence $\theta$ obeys the result and one wants to establish it for $\neg \theta$. $P$ forces $\neg \theta$ if and only if no condition $Q \supseteq P$ forces $\theta$ (by the analogue of Theorem 2.3.4(f)), and since $\theta$ is an $L(Q)$-sentence whenever $Q \supseteq P$ is a condition, the induction hypothesis implies that

$$
P \text { forces } \neg \theta \quad \text { iff } \quad \text { no condition } Q \supseteq P \text { implies } \theta \text {. }
$$

Since $P$ decides all atomic $Q_{F}$-sentences, $P \models \theta$ or $P \models \neg \theta$. If no condition $Q \supseteq P$ implies $\theta, P \forall \theta$ and $P \models \neg \theta$. If $P \models \neg \theta$, then every condition $Q \supseteq P$ implies $\neg \theta$, and since every condition is satisfiable, no condition $Q \supseteq P$ implies $\theta$. Thus $\neg \theta$ obeys the result if $\theta$ does.

The last two results combine to yield the following.

Corollary A.5 If $\psi$ is the $\left(\mathbb{Q}_{F}\right)_{\omega_{1} \omega^{- \text {sentence }}}$

$$
\forall \bar{x} \bigvee_{i \in \mathbb{N}} \exists \bar{y}_{i} \varphi_{i}\left(\bar{x}, \bar{y}_{i}\right)
$$

- where each $\varphi_{i}$ is quantifier-free-and $P$ is a condition, then $P$ forces $\psi$ just in case for all witnesses $\bar{c}$ and conditions $Q \supseteq P$ there are $i \in \mathbb{N}$ and witnesses $\bar{d}_{i}$ for which $T_{\forall}^{\mathcal{Q}} \cup Q \cup\left\{\varphi_{i}\left(\bar{c}, \bar{d}_{i}\right)\right\}$ is satisfiable.

Proof Corollary A.3 implies that $P$ forces $\psi$ just in case for all witnesses $\bar{c}$ and conditions $Q \supseteq P$ there are $i \in \mathbb{N}$, witnesses $\bar{d}_{i}$, and a condition $R \supseteq Q$ that forces $\varphi_{i}\left(\bar{c}, \bar{d}_{i}\right)$. If the condition $R \supseteq P$ forces $\varphi_{i}\left(\bar{c}, \bar{d}_{i}\right)$ for some $i \in \mathbb{N}$ and witnesses $\bar{c}, \bar{d}_{i}$, and $S \supseteq R$ is a condition whose language contains the $c \mathrm{~s}$ and $d \mathrm{~s}$, then $S$ forces $\varphi_{i}\left(\bar{c}, \bar{d}_{i}\right)$, a quantifier-free sentence of $\mathcal{Q}_{L(S)}$, and so $S \models \varphi_{i}\left(\bar{c}, \bar{d}_{i}\right)$ by Lemma A.4 and $T_{\forall}^{\mathcal{Q}} \cup Q \cup\left\{\varphi_{i}\left(\bar{c}, \bar{d}_{i}\right)\right\}$ is satisfied in the $\mathcal{Q}_{L(S)}$-structure with diagram $S$. If, conversely, $Q \supseteq P$ is a condition and $\bar{c}, \bar{d}_{i}$ are witnesses for which $T_{\forall}^{\mathcal{Q}} \cup Q \cup\left\{\varphi_{i}\left(\bar{c}, \bar{d}_{i}\right)\right\}$ is satisfied in $\mathcal{M}$, then since this theory is countable and universal, one may assume that $\mathcal{M}$ is at most countable and has diagram that is a $G$-condition $R \supseteq Q$ for some finite $G \supseteq L(S)$ containing the levels of all the $c$ 's and $d$ 's. Because $\varphi_{i}\left(\bar{c}, \bar{d}_{i}\right)$ is a quantifier-free sentence true in $\mathcal{M}, R \models \varphi_{i}\left(\bar{c}, \bar{d}_{i}\right)$; so $R$ forces $\varphi_{i}\left(\bar{c}, \bar{d}_{i}\right)$ by Lemma A.4.

Specializing the infinitary sentence still further, one may state the next result.

Corollary A.6 If $\psi$ is the $\left(\mathcal{Q}_{F}\right)_{\omega_{1} \omega^{- \text {sentence }}}$

$$
\forall \bar{x} \bigvee_{i \in \mathbb{N}} \exists \bar{y}_{i} \bigwedge_{j \in \mathbb{N}} \varphi_{i j}\left(\bar{x}, \bar{y}_{i}\right)
$$

- where each $\varphi_{i j}$ is a first-order quantifier-free formula - and $P$ is a condition, then $P$ forces $\psi$ just in case for all witnesses $\bar{c}$ and conditions $Q \supseteq P$ there are $i \in \mathbb{N}$ and witnesses $\bar{d}_{i}$ for which every finite subset of $T_{\forall}^{\mathcal{Q}} \cup Q \cup\left\{\varphi_{i j}\left(\bar{c}_{1}, \bar{d}_{i}\right): j \in \mathbb{N}\right\}$ is satisfiable.

Proof By Corollary A.5, $P$ forces $\psi$ just in case for all witnesses $\bar{c}$ and conditions $Q \supseteq P$ there are $i \in \mathbb{N}$ and witnesses $\bar{d}_{i}$ for which $T_{\forall}^{\mathcal{Q}} \cup Q \cup\left\{\bigwedge_{j \in \mathbb{N}} \varphi_{i j}\left(\bar{c}, \bar{d}_{i}\right)\right\}$ is satisfiable; that is, just in case for all witnesses $\bar{c}$ and conditions $Q \supseteq P$ there are 
$i \in \mathbb{N}$ and witnesses $\bar{d}_{i}$ for which $T_{\forall}^{\mathcal{Q}} \cup Q \cup\left\{\varphi_{i j}\left(\bar{c}, \bar{d}_{i}\right): j \in \mathbb{N}\right\}$ is satisfiable. Because the $\varphi_{i j}$ 's are first-order formulas, the desired conclusion follows.

\section{Notes}

1. Any two elements have an upper bound.

2. For any positive integer $n$, if $n x \geq 0$, then $x \geq 0$.

3. If $x, y \leq z, v$, then there is $w$ with $x, y \leq w \leq z, v$.

4. An order unit is a nonnegative element whose natural-number multiples are cofinal in the group.

5. These are the constant symbols used in finite, or "Robinson," forcing with respect to $T^{\mathcal{Q}}$ (see [7, Sections 2.3, 3.4, 4.3]).

6. [6] and [7] rely on different definitions of forcing, but Corollary A.2 of the Appendix shows that their definitions produce the same finite-forcing companion.

7. The length of $c$ is its domain, viewed as a finite ordinal $n=\{0, \ldots, n-1\}$.

8. Though [6, pp. 117-18] does not make exactly the assumptions of this sentence and the next, some or all of these assumptions apparently are made when [6, pp. 168, 237-39] translates the language of second-order arithmetic into others.

9. In what follows, explicit reference to a suitable Gödel numbering is often suppressed.

10. The classes $\Pi_{n}^{0}, \Sigma_{n}^{0}, \Delta_{n}^{0}, \Pi_{n}^{1}, \Sigma_{n}^{1}$, and $\Delta_{n}^{1}$ are defined as in [11, Section 16.1, Exercise 16-8]. The actual quantifier complexity of a prenex formula, in the language of secondorder arithmetic, that defines a set in one of these classes may be slightly higher because not all recursive subsets of $\mathbb{N}^{k} \times \mathcal{P}(\mathbb{N})^{l}$ are represented by atomic formulas in the language used here for second-order arithmetic. However, every recursive relation may be defined by prenex formulas whose prefixes consist of number quantifiers only and may be chosen to be $\forall \exists$ or $\exists \forall$.

11. That is, $S \subseteq \mathbb{N}$ is the set of Gödel numbers of the elements of the graph of a function, from $\mathbb{N}^{3}$ to $\mathbb{N}$, with the given properties.

12. Addison [1] shows that the axiom of constructibility implies the existence of such a well-ordering.

\section{References}

[1] Addison, J. W., "Some consequences of the axiom of constructibility," Fundamenta Mathematicae, vol. 46 (1959), pp. 337-57. Zbl 0091.05301. MR 0124206. 551

[2] Ben Yaacov, I., A. Berenstein, C. W. Henson, and A. Usvyatsov, "Model theory for metric structures," pp. 315-427 in Model Theory With Applications to Algebra and Analysis, Vol. 2, edited by Z. Chatzidakis, D. Macpherson, A. Pillay, and A. Wilkie, vol. 350 of 
London Mathematical Society Lecture Note Series, Cambridge University Press, Cambridge, 2008. MR 2436146. DOI 10.1017/CBO9780511735219.011. 511

[3] Elliott, G. A., "On the classification of inductive limits of sequences of semisimple finitedimensional algebras," Journal of Algebra, vol. 38 (1976), pp. 29-44. Zbl 0323.46063. MR 0397420. 511

[4] Glass, A. M. W., and K. R. Pierce, "Equations and inequations in lattice-ordered groups," pp. 141-71 in Ordered Groups (Boise, Idaho, 1978), edited by J. Smith, G. Kenny, and R. Ball, vol. 62 of Lecture Notes in Pure and Applied Mathematics, Dekker, New York, 1980. MR 0601623. 512, 534

[5] Glass, A. M. W., and K. R. Pierce, "Existentially complete lattice-ordered groups," Israel Journal of Mathematics, vol. 36 (1980), pp. 257-72. Zbl 0454.06007. MR 0597453. DOI 10.1007/BF02762049. 525, 530, 531

[6] Hirschfeld, J., and W. H. Wheeler, Forcing, Arithmetic, Division Rings, vol. 454 of Lecture Notes in Mathematics, Springer, Berlin, 1975. MR 0389581. 512, 525, 527, 530, 531, 534, 535, 543, 544, 547, 548, 549, 551

[7] Hodges, W., Building Models by Games, vol. 2 of London Mathematical Society Student Texts, Cambridge University Press, Cambridge, 1985. MR 0812274. 512, 519, 520, 527, 533, 534, 536, 547, 548, 549, 551

[8] Hodges, W., Model Theory, vol. 42 of Encyclopedia of Mathematics and its Applications, Cambridge University Press, Cambridge, 1993. MR 1221741. DOI 10.1017/CBO9780511551574. 512, 524

[9] Macintyre, A., "A note on axioms for infinite-generic structures," Journal of the London Mathematical Society Second Series, vol. 9 (1974/75), pp. 581-84. Zbl 0308.02055. MR 0363866. 542, 543

[10] Robinson, J., "Definability and decision problems in arithmetic," Journal of Symbolic Logic, vol. 14 (1949), pp. 98-114. Zbl 0034.00801. MR 0031446. 525

[11] Rogers, H., Jr., Theory of Recursive Functions and Effective Computability, McGrawHill, New York, 1967. MR 0224462. 531, 551

[12] Saracino, D., and C. Wood, "Finitely generic abelian lattice-ordered groups," Transactions of the American Mathematical Society, vol. 277 (1983), pp. 113-23. Zbl 0522.06016. MR 0690043. DOI 10.2307/1999347. 512, 526, 534

[13] Scowcroft, P., "Some model-theoretic correspondences between dimension groups and AF algebras," Annals of Pure and Applied Logic, vol. 162 (2011), pp. 755-85. Zbl 1241.03044. MR 2794260. DOI 10.1016/j.apal.2011.02.004. 511

[14] Scowcroft, P., "Existentially closed dimension groups," Transactions of the American Mathematical Society, vol. 364 (2012), pp. 1933-74. MR 2869195. DOI 10.1090/S0002-9947-2011-05382-1. 511, 512, 513, 514, 516, 519, 520, 521, 522, $523,524,527,531,533,534,540,546$

[15] Weispfenning, V., "Model theory of abelian l-groups," pp. 41-79 in Lattice-Ordered Groups: Advances and Techniques, edited by A. M. W. Glass and W. C. Holland, vol. 48 of Mathematics and Applications, Kluwer, Dordrecht, 1989. MR 1036073. 525

\section{Acknowledgments}

I am grateful to Angus Macintyre for asking whether recursive e.c. dimension groups exist. To answer this question I developed the construction used in Theorem 7.4, and in pursuing further applications of this result I came upon the results of Sections 5 through 8 . 
Department of Mathematics and Computer Science Wesleyan University

Middletown, Connecticut, USA

pscowcroft@wesleyan.edu 